Fields Institute Communications

Volume 00, 0000

\title{
Quantifier Elimination for the Relative Frobenius
}

\author{
Thomas Scanlon \\ University of California at Berkeley \\ Department of Mathematics \\ Evans Hall \\ Berkeley, California 94720-3840 \\ USA \\ scanlon@math. berkeley.edu
}

\begin{abstract}
Let $(K, v)$ be a complete discretely valued field of characteristic zero with an algebraically closed residue field of positive characteristic. Let $\sigma: K \rightarrow K$ be a continuous automorphism of $K$ inducing a Frobenius automorphism on the residue field. We prove quantifierelimination for $(K, v, \sigma)$ in a language with angular component maps and in a language with predicates on leading terms. The proof passes through a generalization of the main Ax-Kochen-Ershov and quantifierelimination results of [12] to a wider class of $D$-henselian fields of characteristic zero.
\end{abstract}

\section{Introduction}

A valued difference field is a valued field $(K, v)$ given together with an automorphism $\sigma: K \rightarrow K$ preserving the valuation in the sense that $v x=v \sigma(x)$ holds universally on $K^{\times}$. Examples of valued difference fields are legion though complete fields of positive residue characteristic given together with a relative Frobenius may be the mostly widely exploited.

Valued $D$-fields, generalizations of valued difference and of valued differential fields, were introduced in [12] and quantifier elimination relative to the residue field and the value group for $D$-henselian fields with root-closed linearly differentially closed residue fields of characteristic zero was proved. However, quantifier elimination for valued difference fields eluded the methods of that paper for good reason: if the distinguished automorphism is nontrivial on the residue field, then the theory of $(K, v, \sigma)$ cannot eliminate quantifiers, even relative to the residue field and the value group. The main culprit is the same obstruction to quantifier elimination for (pure) henselian fields with residue fields not closed under roots: the existential quantifier defining the $\ell$-th powers cannot be eliminated simply by expanding the language for the residue field and the value group.

While the formulas defining powers lie at the heart of the failure of quantifier elimination, in order to eliminate quantifiers it does not suffice to simply expand 
the language with power predicates. Fortunately, the solution to the problem for $D$-henselian fields is no more difficult than the solution for henselian (pure) fields.

Ax and Kochen [1] dealt with this difficulty by expanding the language to include a section of the valuation. If $(K, v)$ is a valued field and $\chi: v K \rightarrow K^{\times}$is a section of the valuation, then we may write any element $x \in K^{\times}$as $x=\frac{x}{\chi(v x)} \chi(v x)$. If $K$ is henselian of residue characteristic zero, then $x$ is an $n$-th power if and only if $n$ divides $v x$ and the reduction of $\frac{x}{\chi(v x)}$ is an $n$-th power in the residue field for any $n \in \mathbb{Z}_{+}$.

By expanding the language with a section of the valuation one substantially alters the class of definable sets. Such a section is never definable in the pure valued field language and some of the new definable sets are quite pathological in comparison to those definable in the valued field language. For instance, the image of the section is an infinite definable subset of the field having no interior, while in a henselian field of characteristic zero no set definable in the valued field language can have this property. For some valued fields, notably p-adic fields, it suffices to add power predicates [11], but in general F. -V. Kuhlmann's technology of additive multiplicative congruences (amc structures) is needed to obtain quantifier elimination in a definitional expansion of the valued field language $[2,10]$.

While amc structures meet the condition of not expanding the class of definable sets, they may very well strike the reader as obscure (precise definitions are given in section 4). Angular component functions restore the transparency of the axioms in a language with a cross section while retaining the topological properties of the definable sets in the original language. An angular component function (of level $\mathfrak{m}$ for a pure valued field) is nothing more than a group homomorphism from the units of the field to the units of the residue field which is trivial on the 1-units and which induces the identity on the residue field. If $\pi: \mathcal{O}_{K} \rightarrow \mathcal{O}_{K} / \mathfrak{m}_{K}$ is the residue map and $\chi: v K \rightarrow K^{\times}$is a section of the valuation, then the associated angular component map is $x \mapsto \pi\left(\frac{x}{\chi(v x)}\right)=: \alpha(x)$. For $K$ a henselian field of residue characteristic zero, $x$ is an $n$-th power if and only if $\alpha(x)$ is an $n$-th power and $n$ divides $v x$. We use $\chi$ only to get $\alpha$, the rest of the information supplied by $\chi$ is wasted.

In this work we modify the amc and angular component techniques to suit valued $D$ - fields. We use angular component functions and amc structures of higher level to pass from mixed characteristic to equicharacteristic zero.

The main theorem of this paper is an extension of the results of [12] to a complete axiomatization and quantifier elimination for a wider class of $D$-henselian fields. This class includes all $D$-henselian fields of characteristic zero. Perhaps, the most important example of such a valued $D$-field comes from $\left(W_{p^{\infty}}\left(\mathbb{F}_{p}^{\text {alg }}\right)\left[\frac{1}{p}\right], \sigma_{q}\right)$, the field of fractions of the Witt vectors of $\mathbb{F}_{p}^{\text {alg }}$ (also known as $\widehat{\mathbb{Q}_{p}{ }^{\text {unr }}}$, the completion of the maximal unramified extension of the $p$-adics) with the unique lifting of the $q$-power Frobenius. Independently from the current author, Luc Bélair and Angus Macintyre obtained a version of the main theorem of this paper [4]. Their work and its connections to the present paper will be reported in [5].

This paper is organized as follows. We recall the formalism of amc structures and angular components and adapt them to valued $D$-fields. We then set out the languages to be used and state precisely the theorems to be proved. We present a standard valuation coarsening argument to reduce to the study of valued $D$-fields 
of equicharacteristic zero. The remainder of the paper consists of a detailed guide to modifying the arguments of [12] to the expanded languages.

I originally envisioned the results of the current paper as a part of my thesis [13], but realized through conversations with my thesis advisor, Ehud Hrushovski, that predicates beyond those used in [13] were necessary. A number of people contributed to my understanding of this problem. I thank especially Luc Bélair (for suggesting that angular components may be the most natural framework for quantifier elimination and for pointing out that the angular components are definable in the Witt vectors), Lou van den Dries (for supplying a preprint of [8]), Ehud Hrushovski (for his advice during the work on [13]), Franz-Viktor Kuhlmann (for bringing amc structures to my attention and for his close reading of an earlier version of this paper), and Angus Macintyre (for discussing his work on the relative Frobenius). During the writing of this paper I was partially supported by an NSF MSPRF and NSF Grant DMS-0071890.

\section{Notation and Background}

The notation used in this paper follows that of [12] with the exception that we revert to a more widely used notation for the value group of a valued field and we now write the value of an element $x$ with respect to a valuation $v$ as $v x$ instead of $v(x)$.

We begin this section with our convention on rings and units.

Convention 2.1 For us a ring is a commutative unital ring. If $R$ is a ring, then $R^{\times}:=\{x \in R:(\exists y) x y=1\}$ is the set of units in $R$ considered as subgroup of the multiplicative monoid of $R$.

Notation 2.2 If $K$ is a field with a valuation $v$, then we write write $v x$ for the value of the element $x \in K$. If $x=0$, then we define $v x=\infty$. We write $v K$ for the value group of $K$ and $\mathcal{O}_{K, v}$, or $\mathcal{O}_{K}$ if $v$ is understood, for the ring of integers $\{x \in K: v x \geq 0\}$. The maximal ideal in $\mathcal{O}_{K, v}$ is $\mathfrak{m}_{K, v}=\mathfrak{m}_{K}:=\{x \in K: v x>0\}$. The residue field of $K$ is $k_{K}=k(v)=\mathcal{O}_{K, v} / \mathfrak{m}_{K, v}$. The first of these notations, $k_{K}$, is preferred.

Definition 2.3 A $D$-ring is a commutative ring $R$ given together with a fixed element $e \in R$ and a function $D: R \rightarrow R$ satisfying $D(1)=0, D(x+y)=$ $D(x)+D(y)$, and $D(x \cdot y)=D(x) y+x D(y)+e D(x) D(y)$ universally. On any $D$-ring there is an endomorphism $\sigma: R \rightarrow R$ defined by $x \mapsto e D(x)+x$. The set of $D$-constants, $R^{D}:=\{x \in R: D(x)=0\}$ forms a subring of $R$.

When $e=0$, then the equation relating $D$ to multiplication degenerates to the usual Leibniz rule so that in this case $D$ is nothing other than a derivation. If $e$ is a unit, then $D$ and $\sigma$ are interdefinable.

Definition 2.4 A valued $D$-field is a valued field $(K, v)$ given with a $D$-ring structure for which $v e \geq 0$ and $v D x \geq v x$ holds universally.

If $(R, D, e)$ is a $D$-ring, the ring of $D$-polynomials over $R, R\langle X\rangle_{D}$, is, as a ring the polynomial ring over $R$ in the countably many indeterminates $\left\{D^{j} X\right\}_{j \in \omega}$. The ring of $D$-polynomials has a unique $D$-structure extending that on $R$ with $D\left(D^{j} X\right)=D^{j+1} X$. Given an element $P(X)$ of $R\langle X\rangle_{D}$ we may write $P$ in the form $P(X)=F\left(X, \ldots, D^{d} X\right)$ for some $F\left(X_{0}, \ldots, X_{d}\right) \in R\left[X_{0}, \ldots, X_{d}\right]$. We define $\frac{\partial}{\partial X_{i}} P=\left(\frac{\partial}{\partial X_{i}} F\right)\left(X, \ldots, D^{d} X\right)$. The order of a differential polynomial $P(X), \operatorname{ord} P$, 
is $-\infty$ if $P \in K$ and is the least $d$ such that $P \in K\left[X, \ldots, D^{d} X\right]$ otherwise. If $P(X)=F\left(X, \ldots, D^{d} X\right)$ with $d=\operatorname{ord} P$ then the degree of $P$ is $\operatorname{deg}_{X_{d}} F$. We say that the $D$-polynomial $P$ is simpler than the $D$-polynomial $Q$, written $P \ll Q$, if either $\operatorname{ord} P<\operatorname{ord} Q$ or $\operatorname{ord} P=\operatorname{ord} Q$ and $\operatorname{deg} P<\operatorname{deg} Q$. The total degee of $P$ is the sequence $\left(\operatorname{deg}_{X_{i}} F\right)_{i \in \omega}=: \mathrm{T} \cdot \operatorname{deg}(P)$. We say that a $D$-polynomial of the form $b+\sum_{i=0}^{n} a_{i} D^{i} X$ is affine. If the constant $b$ is zero, then it is linear.

If $L / K$ is an extension of $D$-fields and $a \in L$, then $K(\langle a\rangle)$ is by definition the smallest $D$-subfield of $L$ containing $K$ and $a$.

Recall that a strict henselization of a local ring $(R, \mathfrak{m})$ is an embedding of local rings $\iota: R \hookrightarrow R^{s h}$ for which $R^{s h} / \mathfrak{m} R^{s h}$ is a separably closed field and for which given any other local map $\alpha: R \rightarrow S$ with $S / \mathfrak{m}_{S}$ separably closed, there is a unique (up to automorphism of $R^{s h} / \mathfrak{m}_{R^{s h}}$ ) morphism $\tilde{\alpha}: R^{s h} \rightarrow S$ with $\alpha=\tilde{\alpha} \circ \iota$. We sometimes abuse terminology by referring to the ring $R^{s h}$ as the strict henselization of $R$. For a proof of the existence of the strict henselization see [6].

\section{Multisorted structures}

It is sometimes convenient to regard the value group and residue field of a valued field as separate sorts. Since we use multisorted structures in essential ways later in this paper and this formalism is not so well known even among algebraic model theorists, we recall the definitions in some detail.

Definition 3.1 A multisorted signature $\sigma=(\mathcal{S}, \mathcal{R}, \mathcal{F}, \mathcal{C})$ consists of a nonempty set $\mathcal{S}$ of sort symbols, a set of relation symbols $\mathcal{R}$, a set of function symbols $\mathcal{F}$, and a set of constant symbols $\mathcal{C}$. To each relation symbol $R \in \mathcal{R}$ there is associated a finite sequence of sort symbols, the field of $R, \operatorname{fld}(R)$. Likewise, to each function symbol $f \in \mathcal{F}$ there is associated a finite sequence of sort symbols, the domain of $f, \operatorname{dom}(f)$, and another sort symbol, the range of $f, \operatorname{rng}(f)$. To a constant symbol $c \in \mathcal{C}$ there is an associated sort symbol, the sort of $c, \operatorname{sort}(c)$.

Remark 3.2 A logical signature in the usual sense may be regarded as a multisorted signature by taking $\mathcal{S}$ to consist of a single element.

The multisorted first-order language $\mathcal{L}=\mathcal{L}(\sigma)$ associated to a multisorted signature is constructed from $\sigma$ in much the same way that a usual first-order language is associated to a signature with the crucial exception that each variable is restricted to a single sort (depending on the variable). The following definition make this statement precise.

Definition 3.3 The set $\mathcal{T}(\sigma)$ of $\mathcal{L}(\sigma)$-terms together with the domains and ranges of these terms is defined by the following recursion.

- For each basic sort symbol $S$ and natural number $i \in \omega$, the symbol $x_{i}^{S}$ is an $\mathcal{L}(\sigma)$-term with $\operatorname{dom}\left(x_{i}^{S}\right)=\operatorname{rng}\left(x_{i}^{S}\right)=\langle S\rangle$.

- Each constant symbol $c \in \mathcal{C}$ is an $\mathcal{L}(\sigma)$-term with $\operatorname{dom}(c)=\langle\rangle$, the empty string, and $\operatorname{rng}(c)=\operatorname{sort}(c)$.

- If $f \in \mathcal{F}$ is a function symbol with $\operatorname{dom}(f)=\left\langle S_{1}, \ldots, S_{n}\right\rangle, t_{1}, \ldots, t_{n} \in \mathcal{T}(\sigma)$ are $\mathcal{L}(\sigma)$-terms with $\operatorname{rng}\left(t_{i}\right)=S_{i}$, then $t=f\left(t_{1}, \ldots, t_{n}\right)$ is an $\mathcal{L}(\sigma)$-term with $\operatorname{rng}(t)=\operatorname{rng}(f)$ and $\operatorname{dom}(t)=\operatorname{dom}\left(t_{1}\right) \frown \cdots \frown \operatorname{dom}\left(t_{n}\right)$.

The set $\mathcal{L}(\sigma)$ of $\sigma$-formulas is built from the set of $\sigma$-terms in the usual way. That is, $t=s$ and $R\left(t_{0}, \ldots, t_{n-1}\right)$ are atomic formulas when $t, s, t_{0}, \ldots, t_{n-1}$ are terms and the field of the relation symbol $R$ has field equal to the concatenation 
of the ranges of the $t_{i}$ 's. One then closes under finite Boolean combinations and existential quantification with the proviso that the variable $x_{i}^{S}$ ranges only over the sort $S$. We write $\operatorname{var}(\phi)$ for the set of variables appearing in $\phi$.

I trust that the meaning of multisorted structure is straightforward. The only thing worth remarking is that we require that the sorts partition the universe into disjoint nonempty pieces. If $M$ is a $\sigma$-structure, then there is a surjective function $s: M \rightarrow \mathcal{S}_{\sigma}$ defined by $a \mapsto S$ just in case $a \in S(M)$.

Multisorted languages admit reducts. We say that the multisorted signature $\tau$ is a reduct of the signature $\sigma$ just in case each datum of $\tau$ is a subset of the corresponding datum of $\sigma$. If $\sigma$ is a multisorted signature and $\Sigma \subseteq \mathcal{S}_{\sigma}$ is a nonempty set of sort symbols, then $\sigma \mid \Sigma$ is the reduct of $\sigma$ with $\mathcal{S}_{\sigma \mid \Sigma}=\Sigma, \mathcal{C}_{\sigma \mid \Sigma}:=s^{-1} \Sigma$, $\mathcal{F}_{\sigma \mid \Sigma}:=(\operatorname{dom} \times \operatorname{rng})^{-1}\left(<^{\omega} \Sigma \times \Sigma\right)$, and $\mathcal{R}_{\sigma \mid \Sigma}:=\mathrm{fld}^{-1} \Sigma$.

If $M$ is a $\sigma$-structure and $\Sigma \subseteq \mathcal{S}_{\sigma}$ is a non-empty set of sorts, then $M \uparrow \Sigma:=$ $\pi^{-1} \Sigma$ is the reduct of $M$ to $\sigma\lceil\Sigma$.

We could continue by laying out the meaning for multisorted languages of other fundamental logical concepts, but we trust that the reader can supply the missing definitions.

With the foundations of multisorted languages in place we can now say precisely what is meant by relative quantifier elimination and relative completeness.

Let $\sigma$ be a multisorted signature and $\Sigma \subseteq \mathcal{S}_{\sigma}$ a set of sort symbols. We define a new signature $\sigma^{\prime}:=\sigma_{q f-\Sigma}$ having the same constant and function symbols and $\mathcal{R}_{\sigma^{\prime}}:=\mathcal{R}_{\sigma} \cup\left\{R_{\phi}: \phi \in \mathcal{L}(\sigma), \operatorname{var}(\phi) \subseteq\left\{x_{S}^{(i)}: S \in \Sigma, i \in \omega\right\}\right\}$. Fixing an ordering of the free variables of $\phi, x_{S_{0}}^{\left(i_{0}\right)}, \ldots, x_{S_{m-1}}^{i_{m-1}}$, we define the field of $R_{\phi}$ to be $\left\langle S_{0}, \ldots, S_{m-1}\right\rangle$. The theory $T_{q f-\Sigma, \sigma}$ is generated by the (universal closures of) the formulas $\phi \leftrightarrow R_{\phi}$ for $\phi \in \mathcal{L}(\sigma)$ with $\operatorname{rng}(\operatorname{var}(\phi)) \subseteq \Sigma$.

Definition 3.4 Let $\sigma$ be a multisorted signature, $\Sigma \subseteq \mathcal{S}_{\sigma}$ a set of $\sigma$-sort symbols, and $T$ an $\mathcal{L}(\sigma)$-theory.

We say that $T$ is complete relative to $\Sigma$ if for any model $M \models T$ the theory $T \cup \operatorname{Th}_{\mathcal{L}(\sigma \mid \Sigma)}(M \uparrow \Sigma)$ is complete. We say that $T$ eliminates quantifiers relative to $\Sigma$ if for any nonsentence $\varphi \in \mathcal{L}(\sigma)$ there is a quantifier-free formula $\psi \in \mathcal{L}\left(\sigma_{q f-\Sigma}\right)$ such that $T \cup T_{q f-\Sigma, \sigma} \vdash \varphi \leftrightarrow \psi$.

We intend to prove not only relative completeness and quantifier elimination results in a fixed language but rather such results for any expansion of the language of valued difference fields by structure on the mixed structures.

Definition 3.5 Let $\sigma$ be a multisorted signature, $\Sigma \subseteq \mathcal{S}_{\sigma}$ be a nonempty set of sorts, and $T$ an $\mathcal{L}(\sigma)$-theory. We say that $T$ is respendently complete relative to $\Sigma$ if for any model $M \models T$ and any signature $\tau \supseteq(\sigma \mid \Sigma)$ with $\mathcal{S}_{\tau}=\Sigma$ and any expansion $M^{\prime}$ of $(M \uparrow \Sigma)$ to a $\tau$-structure, $T \cup \operatorname{Th}_{\mathcal{L}(\tau)}\left(M^{\prime}\right)$ is complete. Likewise, $T$ resplendently eliminates quantifiers relative to $\Sigma$ if for any $\tau$ and $M^{\prime}$ as above and any nonsentence $\phi \in \mathcal{L}(\sigma \cup \tau)$, there is some quantifier-free $\psi \in \mathcal{L}\left((\sigma \cup \tau)_{q f-\Sigma}\right)$ such that $T \cup T_{q f-\Sigma, \sigma \cup \tau} \vdash \phi \leftrightarrow \psi$.

Recall that a definable set $X$ is stably embedded in the structure $M$ if for every elementary extension $M^{*} \succeq M$, if $X^{*}$ is the interpretation of $X$ in $M^{*}, n \in \omega$, and $D \subseteq\left(M^{*}\right)^{n}$ is definable (with parameters), then $D \cap\left(X^{*}\right)^{n}$ is definable in the reduct $X^{*}$. With the same definition we extend this notion to the case that $X$ is a union of sorts. 


\section{Leading Terms and Angular Components}

In this section we recall the formalism of additive multiplicative congruences, or what we prefer to call leading terms, and of angular component functions. As is the case for Henselian fields, reasonable versions of completeness and quantifier elimination hold for valued $D$-fields relative to the leading terms.

Definition 4.1 If $(K, v)$ is a valued field and $I \subset \mathcal{O}_{K, v}$ is a proper ideal, then the group of I-leading terms is the quotient group $K_{I}:=K^{\times} /(1+I)$.

As the ideals of a valuation ring are linearly ordered by inclusion, there are natural quotient maps between the leading terms for different ideals.

Definition 4.2 If $(K, v)$ is a valued field and $I \subseteq J \subset \mathcal{O}_{K, v}$ are proper ideals, then we denote the natural projection map by $\pi_{I, J}: K_{I} \rightarrow K_{J}$. In the case that $I=(0)$, we identify $K_{I}$ with $K^{\times}$and write merely $\pi_{J}: K^{\times} \rightarrow K_{J}$.

Of course, the $I$-leading terms carry more structure than merely multiplication. For example, the valuation on $K^{\times}$descends to $K_{I}$. We continue to denote the valuation on $K_{I}$ by $v$. Addition does not descend to a well-defined binary operation, but it does induce a ternary relation.

Definition 4.3 If $(K, v)$ is a valued field and $I, J \subset \mathcal{O}_{K, v}$ are proper ideals, then addition on $K$ induces a ternary relation $A_{I, J}:=\left\{(x, y, z) \in K_{I} \times K_{I} \times\right.$ $\left.K_{J} \mid\left(\exists \tilde{x}, \tilde{y} \in K^{\times}\right) \pi_{I}(\tilde{x})=x \& \pi_{I}(\tilde{y})=y \& \pi_{J}(\tilde{x}+\tilde{y})=z\right\}$.

While addition on the leading terms is not a function, it is not quite as badly behaved as one might expect. For example, if $I=\mathfrak{m}_{K} \subset \mathcal{O}_{K}$ is the maximal ideal of the valuation ring of a valued field, then if $x, y \in K^{\times}$and $v(x+y)=v(x)=v(y)$, then $\pi_{I}(x+y)$ is the unique element of $A_{I, I}\left(\pi_{I}(x), \pi_{I}(y), K_{I}\right)$. In this case we abuse notation and write $x+y$ for this unique element.

When dealing with a valued $D$-field $(K, v, D, e)$, one should also consider the extra structure on the leading terms induced by $D$ and $\sigma$.

Since $\sigma$ respects the valuation, it induces a function on $K_{I}$ for any ideal $I \subset \mathcal{O}_{K}$. We continue to denote this function by $\sigma$. The function $D: K \rightarrow K$ does not induce a function on the leading terms. Instead, we have only binary relations.

Definition 4.4 If $(K, v, D, e)$ is a valued $D$-field and $I, J \subset \mathcal{O}_{K}$ are proper ideals, then $D_{I, J}$ is the binary relation $\left\{(x, y) \in K_{I} \times K_{J} \mid\left(\exists \tilde{x} \in \pi_{I}^{-1}\{x\}\right) \pi_{J}(D \tilde{x})\right\}$.

As with addition, $D_{I, J}$ is not that far from being a function. Again, we abuse notation writing $D_{I, J}(x)$ for the unique $y$ satisfying $(x, y) \in D_{I, J}$.

We use the leading terms technology only in the case of certain definable ideals.

Definition 4.5 Let $(K, v)$ be a valued field. Let $p:=\operatorname{char}(k(v))$ if the residue characteristic is positive and set $p:=1$ otherwise. For each natural number $n$ define $I_{n}:=\left\{x \in \mathcal{O}_{K} \mid v x>n v p\right\}$. Denote $K_{I_{n}}$ by $K_{n}$. We write $R_{n}$ for $\mathcal{O}_{K} / I_{n}$.

Note that in equicharacteristic zero, the ideals $I_{n}$ are all equal to $\mathfrak{m}_{K}$.

The ideals $I_{n}$ are all definable in the language of valued fields. Thus, the leading terms $K_{n}$ are interpretable already in the language of valued fields.

We note that the ring structure on $R_{n}$ is interpretable in $K_{n}$. Set $\left(\mathcal{O}_{K}\right)_{n}:=$ $\left\{x \in K_{n}: v(x) \geq 0\right\}$. Define an equivalence relation on $\left(\mathcal{I}_{K}\right)_{n}$ by $x \sim y \Leftrightarrow$ $(\exists z)\left[A_{n, n}(x,-y, z) \rightarrow v(z)>n v p\right]$. As a set, $R_{n}$ is $\left(\mathcal{O}_{K}\right)_{n} / \sim$. It follows immediately from the definitions that multiplication on $K_{n}$ induces the usual multiplication on $R_{n}$ and the addition defined by $[x]_{\sim}+[y]_{\sim}=[z]_{\sim} \leftrightarrow A_{n, n}(x, y, z)$ is the usual 
addition on $R_{n}$. Likewise, $D[x]_{\sim}=[y]_{\sim} \leftrightarrow D_{n, n}(x, y)$ defines the usual $D$-ring structure on $R_{n}$.

For a sufficiently saturated valued field $K$, the valuation exact sequence

$1 \longrightarrow\left(\mathcal{O}_{K} / I\right)^{\times} \longrightarrow K_{I} \longrightarrow v K \longrightarrow 0$

splits. If there were a definable splitting, then the structure on $K_{I}$ could be understood entirely in terms of the structure on the residue ring $\mathcal{O}_{K} / I$ and on the value group. In many valued fields, no such splitting is definable in the language of valued fields, but one can work with expanded languages having function symbols for these splittings.

Definition 4.6 Let $(K, v)$ be a valued field and $I \subseteq \mathcal{O}_{K, v}$ a proper ideal. An angular component function of level $I$ is a section $\mathrm{ac}_{I}: K_{I} \rightarrow\left(\mathcal{O}_{K} / I\right)^{\times}$of the valuation exact sequence

$$
1 \longrightarrow\left(\mathcal{O}_{K} / I\right)^{\times} \longrightarrow K_{I} \longrightarrow v K \longrightarrow 0
$$

We sometimes abuse notation and write $\mathrm{ac}_{I}$ for $\mathrm{ac}_{I} \circ \pi_{I}$.

If $\mathcal{I}$ is a family of proper ideals of $\mathcal{O}_{K, v}$, then we say that $\left\{\operatorname{ac}_{I} \mid I \in \mathcal{I}\right\}$ forms a family of angular component function if for each $I$ the function $\mathrm{ac}_{I}$ is an angular component function of level $I$ and if for each pair $I \subseteq J \in \mathcal{I}$, we have $\mathrm{ac}_{J} \circ \pi_{I, J}=\pi_{I, J} \circ \mathrm{ac}_{I}$.

Just as we only consider leading terms for the ideals $I_{n}=\left\{x \in \mathcal{O}_{K} \mid v x>n v p\right\}$, we only consider angular components relative to these ideals. We write $\mathrm{ac}_{n}$ for $\mathrm{ac}_{I_{n}}$.

Angular component functions respect addition weakly. More precisely, we have the following lemma.

Lemma 4.7 If $(K, v)$ is a valued field, $I \subset \mathcal{O}_{K}$ is a proper ideal, $\mathrm{ac}_{I}: K^{\times} \rightarrow$ $\left(\mathcal{O}_{K} / I\right)^{\times}$is an angular component function of level $I$, and $x, y \in K^{\times}$with $v(x+y)=$ $v x=v y$, then $\mathrm{ac}_{I}(x+y)=\mathrm{ac}_{I}(x)+\mathrm{ac}_{I}(y)$.

Proof Since $\mathrm{ac}_{I}$ is a section, if $x \in K^{\times}$and $\pi_{I}(x) \in\left(\mathcal{O}_{K} / I\right)^{\times}$, then we have $\operatorname{ac}_{I}(x)=\pi_{I}(x)$. From this observation we compute:

$$
\begin{aligned}
\operatorname{ac}_{I}(x+y) & =\operatorname{ac}_{I}(x) \operatorname{ac}_{I}\left(1+\frac{y}{x}\right) \\
& =\operatorname{ac}_{I}(x) \pi_{I}\left(1+\frac{y}{x}\right) \\
& =\operatorname{ac}_{I}(x)\left(1+\pi_{I}\left(\frac{y}{x}\right)\right) \\
& =\mathrm{ac}_{I}(x)+\mathrm{ac}_{I}(x) \pi_{I}\left(\frac{y}{x}\right) \\
& =\mathrm{ac}_{I}(x)+\mathrm{ac}_{I}(x) \mathrm{ac}_{I}\left(\frac{y}{x}\right) \\
& =\mathrm{ac}_{I}(x)+\mathrm{ac}_{I}(y)
\end{aligned}
$$

However, if $v(x+y) \neq v x$ and $v(x+y) \neq v y$, then we can say nothing about $\operatorname{ac}_{I}(x+y)$ in terms of $\operatorname{ac}_{I}(x)$ and $\operatorname{ac}_{I}(y)$.

If $(K, v, D, e)$ is a valued $D$-field, then recall that $\sigma: K \rightarrow K$ defined by $\sigma(x):=e D(x)+x$ is an endomorphism of $K$. If $v(e)>0$, then $\sigma$ is necessarily an automorphism and $v x=v \sigma(x)$ holds for all $x \in K^{\times}$. However, if $v(e)=0$, the endomorphism $\sigma$ need be neither surjective nor valuation preserving. 
Convention 4.8 From now on we include as part of the definition of a valued $D$-field that $\sigma$ is a valuation preserving automorphism.

If $K$ is a valued $D$-field and $\mathcal{I}$ is a family of ideals in $\mathcal{O}_{K}$, then a system of $D$-field angular component functions for $\mathcal{I}$ is defined to be a system of angular component functions for $\mathcal{I}$ in the sense of pure valued fields which respect the $D$ - structure. That is, $\operatorname{ac}_{J}\left(D_{I, J}(x)\right)=\pi_{I, J}\left(D \operatorname{ac}_{I}(x)\right)$ for $x$ with $v D x=v x$ and $\operatorname{ac}_{I}(\sigma(x))=\sigma\left(\operatorname{ac}_{I}(x)\right)$ for all $x$.

From an angular component function $\mathrm{ac}_{I}: K_{I} \rightarrow\left(\mathcal{O}_{K} / I\right)^{\times}$we obtain a section of the valuation $\chi_{I}: \Gamma_{K} \rightarrow K_{I}$ by the formula $\chi_{I}(\gamma):=\frac{x}{\mathrm{a}_{I}(x)}$ for any choice of $x \in K_{I}$ with $v x=\gamma$.

Lemma 4.9 Let $(K, 0,1, e,+, \cdot, D, v)$ be a valued $D$-field, $I \subset \mathcal{O}_{K}$ a proper ideal, $\mathrm{ac}_{I}: K_{I} \rightarrow\left(\mathcal{O}_{K} / I\right)^{\times}$an angular component function of level $I$, and $\chi_{I}:$ $\Gamma_{K} \rightarrow K_{I}$ the corresponding section on the value group. Then the angular component function $\mathrm{ac}_{I}$ respects the $D$ structure if the range of $\chi_{I}$ is contained in the set of $D$-constants, the set $\left\{x \in K_{I}:\left(\forall x^{\prime} \in K^{\times}\right) \pi\left(x^{\prime}\right)=x \rightarrow \frac{D x^{\prime}}{x^{\prime}} \in I\right\}$. The converse is true as long as $D$ is non-trivial on the residue field.

Proof We show first that $\operatorname{ac}_{I}$ respect the $D$-structure if $\chi_{I}$ takes values in the $D$-constants. Let $x \in K_{I}$ and suppose that $D_{I, I}(x)$ is defined. Write $x=$ $\operatorname{ac}_{I}(x) \chi_{I}(v x)$. Let $a, b \in K^{\times}$with $\pi_{I}(a)=\operatorname{ac}_{I}(x)$ and $\pi_{I}(b)=\chi_{I}(v x)$. Then by the twisted Leibniz rule we have $D(a b)=a D(b)+\sigma(b) D(a)$. As $\chi_{I}$ takes values in the $D$-constants (modulo $1+I$ ), we have $D(b) \in b I$ and $\sigma(b) \in b(1+I)$. So we have $D(a b)=b D(a)+b D(a) i+a b i^{\prime}$ for some $i, i^{\prime} \in I$. As $K$ is a valued $D$ field, we have $v(b D(a)) \geq v(a b)$. Thus, the hypothesis that $v x=v D x$ implies that $v(D a)=v(a)$. Thus, we have $D(a b) \equiv b D(a)(1+I)$. Applying $\pi_{I}$, we have $D(x)=$ $\chi_{I}(v x) D\left(\operatorname{ac}_{I}(x)\right)$. As $v x=v(D(x))$, we have $\chi_{I}(D(x))=\chi_{I}\left(v(D(x)) D\left(\operatorname{ac}_{I}(x)\right)\right.$. As generally we have $\mathrm{ac}_{I}(y)=\frac{y}{\chi_{I}(v y)}$, we conclude that $D\left(\mathrm{ac}_{I}(x)\right)=\mathrm{ac}_{I}(D x)$.

For the other implication, let $\gamma \in \Gamma_{K}$.

The hypothesis that $D$ is non-trivial on the residue field implies that we can find some $x \in K^{\times}$with $v x=\gamma=v D x$. That is, choose any $y$ with $v y=\gamma$. If $y$ does not already work, then look for some $\alpha$ with $v(\alpha)=0$ and $\gamma=v(D(\alpha y))=$ $v(D(\alpha) y+\sigma(\alpha) D(y))$. As $v(D(y))>\gamma$ and $v(\alpha)=0$, we achieve this is $v(D(\alpha))=0$.

For this choice of $x$, write $x=\operatorname{ac}_{I}(x) \chi_{I}(\gamma)$. As $v x=v D x$ we have $D(x)=$ $\operatorname{ac}_{I}(D x) \chi_{I}(\gamma)$ and $D(x)=D\left(\operatorname{ac}_{I}(x)\right) \chi_{I}(\gamma)+\sigma\left(\operatorname{ac}_{I}(x)\right) D\left(\chi_{I}(\gamma)\right)=\mathrm{ac}_{I}(D x) \chi_{I}(\gamma)+$ $\sigma\left(\operatorname{ac}_{I}(x)\right) D\left(\chi_{I}(\gamma)\right)$. As $v\left(\sigma\left(\operatorname{ac}_{I}(x)\right)\right)=v\left(\operatorname{ac}_{I}(x)\right)=0$, we conclude that $\frac{D\left(\chi_{I}(\gamma)\right)}{\chi_{I}(\gamma)} \in I$ as claimed.

\section{Languages}

In this section we explain how valued $D$-fields are to be considered as multisorted structures. The mutlisorted signature of valued difference fields, $\tau_{v d f}$ is defined as follows. There are sort symbols $K, \Gamma, k$, and $K_{n}$ and $R_{n}$ for each $n \in \omega$. There are function symbols $+; \cdot ; \sigma ; v ;+_{\Gamma} ; \overline{+}, \bar{\cdot}$, and $\bar{\sigma} ;{ }_{n}, \pi_{n}, v_{n}, \sigma_{n}$, and $\pi_{n, 0}$ (for $n \in \omega), \pi_{n, m}$. There are relation symbols $<$ and $A_{n}$ for $n \in \omega$. There are constant symbols $0,1, p, 0_{\Gamma}, \infty, 0_{n}, 1_{n}$, and $p_{n}$ (for $n \in \omega$ ), $\overline{0}, \overline{1}, \bar{p}$, and $\bar{\infty}$. The following table summarizes the relations between these symbols and the sorts. 


\begin{tabular}{|c|c|c|c|c|c|c|}
\hline $\mathcal{F}$ & dom & rng & $\mathcal{C}$ & sort & $\mathcal{R}$ & fld \\
\hline+ & $K \times K$ & $K$ & 0 & $K$ & $<$ & $\Gamma \times \Gamma$ \\
\hline . & $K \times K$ & $K$ & 1 & $K$ & $A_{n}$ & $K_{n} \times K_{n} \times K_{n}$ \\
\hline$\pi$ & & $k$ & $p$ & $K$ & $D_{n}$ & $K_{n} \times K_{n}$ \\
\hline$\sigma$ & $K$ & $K$ & $0_{n}$ & $K_{n}$ & & \\
\hline$+_{\Gamma}$ & $\Gamma \times \Gamma$ & $\Gamma$ & $1_{n}$ & $K_{n}$ & & \\
\hline$\pi_{n}$ & $K$ & $K_{n}$ & $p_{n}$ & $K_{n}$ & & \\
\hline$v$ & $K$ & $\Gamma$ & $0_{\Gamma}$ & $\Gamma$ & & \\
\hline$v_{n}$ & $K_{n}$ & $\Gamma$ & $\infty_{\Gamma}$ & $\Gamma$ & & \\
\hline$\pi_{n, m}$ & $K_{n}$ & $K_{m}$ & $\overline{0}$ & $k$ & & \\
\hline$\pi_{n, 0}$ & $K_{n}$ & $\mathrm{k}$ & $\overline{1}$ & $k$ & & \\
\hline$\sigma_{n}$ & $K_{n}$ & $K_{n}$ & $\bar{p}$ & $k$ & & \\
\hline $\bar{\tau}$ & $k \times k$ & $k$ & $\bar{\infty}$ & $k$ & & \\
\hline - & $k \times k$ & $k$ & & & & \\
\hline $\bar{\sigma}$ & $k$ & $k$ & & & & \\
\hline
\end{tabular}

In principle, we should explicitly indicate the structure on $R_{n}$, but we will regard the $\mathcal{L}_{D-\text { ring }}\left(\sigma, \sigma^{-1}\right)$-structure $R_{n}$ as interpretable in $K_{n}$.

We write $\mathcal{L}_{\text {vdf }}$ for $\mathcal{L}\left(\tau_{v d f}\right)$. If $(L, v, \sigma)$ is a valued difference field with $q \in \mathcal{O}_{L}$, then we interpret $\mathcal{L}_{\mathrm{vdf}}$ as indicated in the following table. 


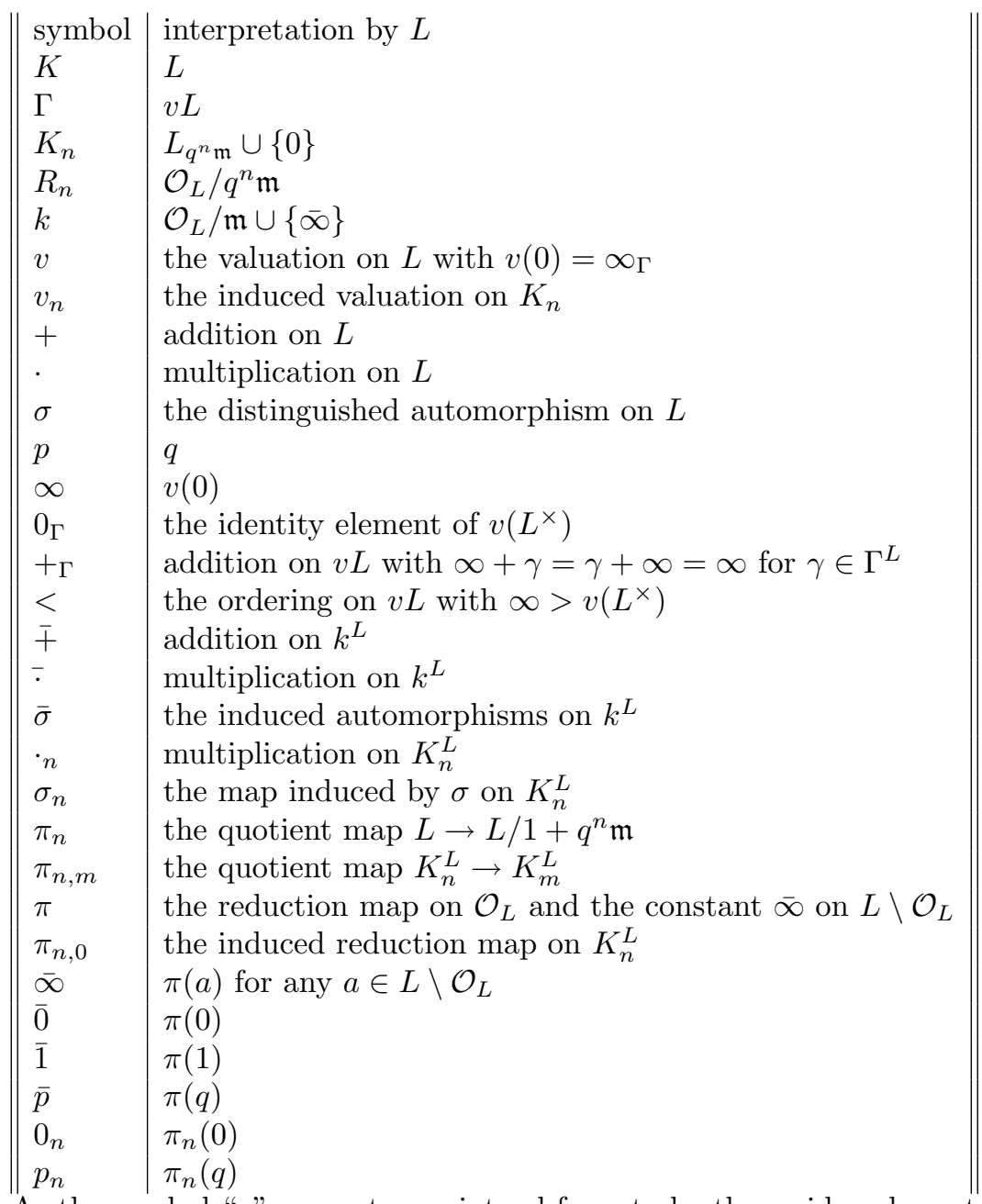

As the symbol " $p$ " suggests, we intend for $p$ to be the residue characteristic in the case of positive residue characteristic and 1 when the residue characteristic is zero. In the case that $p^{L}$ is a unit, the maps $\pi_{n, m}: K_{n}^{L} \rightarrow K_{m}^{L}$ are isomorphisms so that there is no need to go beyond the reduct to $\left(K, \Gamma, K_{0}\right)$. In order to make a uniform statement, we do not explicitly specify the value of $p$. However, in mixed characteristic, we require that $v p^{L}$ is at least that of $p$, the residue characteristic.

We obtain the language of valued difference fields with angular components, $\mathcal{L}_{\mathrm{vdf}}^{\mathrm{ac}}$, by adjoining to $\mathcal{L}_{\mathrm{vdf}}$ function symbols $\left\{\mathrm{ac}_{n}\right\}$ having domain and range sort $K_{n}$.

\section{Axioms}

The class of valued $D$-fields considered in this paper is larger than that of [12] due to the relaxation of three conditions on the residue field. First, we no longer insist that the residue characteristic is zero. Secondly, we now allow for the possibility of $v e=0$. Finally, we do not demand that the residue field be closed under roots. We require only that the residue field be linearly $D$-closed. As shown in [12], this is an intrinsic property of $D$-henselian fields. We also insist on generic characteristic zero. It ought to be possible to relax this last condition as well, but given the 
current state of knowledge about the model theory of pure valued fields of positive characteristic, I expect this would take significantly new methods.

The axioms for valued $D$-fields given in [12] do not suffice for the more general valued $D$-fields we consider here. Since the language has been expanded and the conditions on the residue field relaxed, we need to modify the axioms for valued $D$-fields. In stating the axioms, we give the axioms for valued $D$-fields in the basic language of valued $D$-fields, $\mathcal{L}_{\text {vdf }}$.

The first three axioms describe valued $D$-fields.

Axiom $1 K$ is $D$-field, $k$ is a $D$-field and $\Gamma$ is an ordered abelian group. [NB: we require a $D$-field to be an $\mathcal{L}_{\mathrm{D}-\text {-ring }}\left(\sigma, \sigma^{-1}\right)$-structure in which $\sigma$ is an automorphism with inverse $\sigma^{-1}$ satisfying the equation $\sigma=e D+\mathrm{id}$.]

Axiom 2 The inequality $v D x \geq v x$ holds universally as does the equality $v x=v \sigma(x)$.

Axiom $3 K$ is a valued field with value group a subgroup of $\Gamma$ via $v$ and residue field a subfield of $k$ via $\pi$. The map $\pi$ restricted to $\mathcal{O}_{K}$ is a map of $\mathcal{L}_{\mathrm{D}-\text { ring }}\left(\sigma, \sigma^{-1}\right)$ structures. The maps $\pi_{n}: K^{\times} \rightarrow K_{n}$ express $K_{q^{n} \mathfrak{m}}$ as a substructure of $K_{n}$.

The next there axioms finish the description of $D$-henselian fields.

Axiom 4 The maps $\pi, \pi_{n}$ (for $n \in \omega$ ) and $v$ are surjective.

Axiom $5 K$ has enough constants: $v\left(\left(K^{D}\right)^{\times}\right)=\Gamma$. That is, for any $\gamma \in \Gamma$ there is some $x \in K$ with $D x=0$ and $v x=\gamma$.

Axiom 6 D-Hensel's Lemma: If $P(X) \in \mathcal{O}_{K}\langle X\rangle_{D}$ and $a \in \mathcal{O}_{K}$ with $\left.v(P(a))\right\rangle$ $0=v\left(\frac{\partial}{\partial X_{i}} P(a)\right)$ for some non-negative integer $i$, then there is some $b \in \mathcal{O}_{K}$ with $P(b)=0$ and $v(a-b)>0$.

A valued $D$-field with an angular component function (of level zero) is a valued $D$-field given together with an angular component function $\mathrm{ac}_{0}: K_{0} \rightarrow k$ of level $\mathfrak{m}$. Likewise, a valued $D$-field with a system of angular component functions is an expansion of a valued $D$-field to the language $\mathcal{L}_{\text {vdf }}^{\mathrm{ac}}$ in which the angular component function symbols are interpreted as a system of angular components for $\left\{I_{n}:=\{x \in\right.$ $\left.\left.\mathcal{O}_{K}: v x>n v(p)\right\}\right\}_{n \in \omega}$.

Remark 6.1 In [12] the axioms for a valued $D$-field are given relative to a fixed theory of the residue field and value group. We present a semantic version of that completeness result below. The syntactic form is somewhat more complicated for mixed characteristic valued $D$ - fields. In particular, it does not suffice to specify the theory of the residue field and of the value group in order to give a completion of the theory of $D$-henselian fields.

Remark 6.2 Proposition 5.3 of [12] applies to the modified axioms for $D$ henselian fields also. Thus, if $K$ is a $D$-henselian field, its residue field, $k$, is linearly $D$-closed.

We can now state our main theorem. Actually, we will state two different versions, one in $\mathcal{L}_{\text {vdf }}^{\text {ac }}$ and on in $\mathcal{L}_{\text {vdf }}$.

Theorem 6.3 The theory of D-henselian fields of characteristic zero in the language $\mathcal{L}_{\mathrm{vdf}}^{\mathrm{ac}}$ together with a consistent atomic diagram is resplendently complete and resplendently eliminates quantifiers relative to the value group and the residue rings $R_{n}(n \in \omega)$. 
The version of the theorem in $\mathcal{L}_{\mathrm{vdf}}$ is almost the same.

Theorem 6.4 The theory of D-henselian fields of characteristic zero in the language $\mathcal{L}_{\mathrm{vdf}}$ together with a consistent atomic diagram is resplendently complete and resplendently eliminates quantifiers relative to the leading terms $K_{n}(n \in \omega)$.

\section{From angular components to leading terms}

In this section we show our theorem stated in $\mathcal{L}_{\mathrm{vdf}}$ follows formally from the theorem stated in $\mathcal{L}_{\mathrm{vdf}}^{\mathrm{ac}}$.

We start with some general results on relative quantifier elimination.

Lemma 7.1 If $\sigma$ is a multisorted signature, $\Sigma \subseteq \mathcal{S}_{\sigma}$ is a nonempty set of sorts, and $T$ is an $\mathcal{L}(\sigma)$-theory which eliminates quantifiers relative to $\Sigma$, then for any $M \models T$ the reduct $(M \uparrow \Sigma)$ is stably embedded if and only if every $\mathcal{L}(\sigma)$-quantifier free definable set in $(M \uparrow \Sigma)$ is $\mathcal{L}(\sigma \nmid \Sigma)$-definable.

Proof Let $M \models T$ be a model of $T, \phi(x, y)$ be an $\mathcal{L}(\sigma)$ formula with $x$ a tuple of variables ranging over sorts in $\Sigma$ and $y$ another tuple of variables, and $a$ be any parameter from $M$ substitutable for $y$. We are charged with finding some $\mathcal{L}(\sigma \mid \Sigma)_{M\lceil\Sigma}$-formula $\eta(x)$ for which $M \models(\forall x)[\eta(x) \leftrightarrow \phi(x, a)]$.

By our hypothesis that $T$ eliminates quantifiers relative to $\Sigma$, there is some quantifier-free $\mathcal{L}\left(\sigma_{q f-\Sigma}\right)$-formula $\theta(x, y)$ such that

$$
T \cup T_{\mathrm{qf}-\Sigma, \sigma} \vdash \theta(x, y) \leftrightarrow \phi(x, y)
$$

That is, there are a quantifier-free $\mathcal{L}(\sigma)$-formula $\vartheta(x, y)$ and an $\mathcal{L}(\sigma)$-formula $\psi(x, y)$ having $\operatorname{var}(\psi) \subseteq\left\{x_{i}^{(S)}: i \in \omega, S \in \Sigma\right\}$ with

$$
T \vdash(\forall x, y)[\psi(x, y) \leftrightarrow \vartheta(x, y) \wedge \psi(x, y)]
$$

By our hypothesis that every quantifier-free $\mathcal{L}(\sigma)_{M}$-definable set in $\Sigma(M)$ is $\mathcal{L}(\sigma$ ।

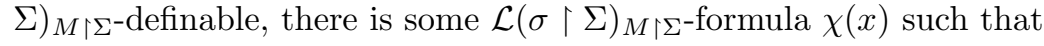

$$
T \vdash \chi(x) \leftrightarrow \vartheta(x, a)
$$

As the formula $\psi(x, y)$ contains only variables ranging over sorts in $\Sigma$, even though some of the sorts of the parameter $a$ may lie outside of $\Sigma$, the formula $\psi(x, a)$ is actually an $\mathcal{L}\left(\sigma\lceil\Sigma)_{M \uparrow \Sigma}\right.$-formula. Thus the formula $\eta(x):=\chi(x) \wedge \psi(x, a)$ is an $\mathcal{L}(\sigma \mid \Sigma)_{M \uparrow \Sigma}$-formula and

$$
M \models(\forall x)[\phi(x, a) \leftrightarrow \eta(x)]
$$

Lemma 7.2 Let $\sigma \subseteq \sigma^{\prime}$ be a multisorted signatures. Let $\Sigma \subseteq \mathcal{S}_{\sigma}$ be a set of sort symbols. We suppose that the only difference between $\sigma$ and $\sigma^{\prime}$ is that there may be new function symbols in $\sigma^{\prime}$. Let $T$ be a $\mathcal{L}(\sigma)$-theory and $T^{\prime} \supseteq T$ a a $\mathcal{L}\left(\sigma^{\prime}\right)$ theory which eliminates quantifiers in $\mathcal{L}\left(\sigma^{\prime}\right)$ and with $T=T^{\prime} \uparrow \mathcal{L}(\sigma)$.

We make the following assumptions.

- If $f \in \mathcal{F}_{\sigma^{\prime}} \backslash \mathcal{F}_{\sigma}$, then $\operatorname{dom}(f) \in{ }^{<\omega} \Sigma$ and $\operatorname{rng}(f) \in \Sigma$.

- If $f \in \mathcal{F}_{\sigma}$ and $\operatorname{rng}(f) \in \Sigma$, then there are a function symbol $\tilde{f} \in \mathcal{F}_{\sigma}$ and $\sigma$-terms $h_{0}, \ldots, h_{m-1} \in \mathcal{T}(\sigma)$ such that $\operatorname{dom}(\tilde{f}) \in{ }^{<\omega} \Sigma$ and $T \vdash f=\tilde{f} \circ$ $\left\langle h_{0}, \ldots, h_{m-1}\right\rangle$.

- If $R \in \mathcal{R}_{\sigma}$, then either $\operatorname{fld}(R) \in{ }^{<\omega} \Sigma$ or $\operatorname{fld}(R) \in \in^{<\omega}\left(\mathcal{S}_{\sigma} \backslash \Sigma\right)$.

- If $t \in \mathcal{T}(\sigma)$ is a $\sigma$-term with $\operatorname{var}(t) \subseteq\left\{x_{S}^{(i)}: S \in \Sigma, i \in \omega\right\}$, then $\operatorname{rng}(t) \in \Sigma$. 
If $\Sigma$ is stably emebedded (relative to $T$ ), then $T$ eliminates quantifiers in $\mathcal{L}(\sigma)$ relative to $\Sigma$.

Proof Let $\phi \in \mathcal{L}(\sigma)$ be a nonsentence. We are charged with finding a quantifierfree $\mathcal{L}\left(\sigma_{\mathrm{qf}-\Sigma}\right)$-formula $\eta$ such that

$$
T \cup T_{\mathrm{qf}-\Sigma, \sigma} \vdash \phi \leftrightarrow \eta
$$

By our hypothesis that $T^{\prime}$ eliminates quantifiers, there is some quantifier-free $\psi \in \mathcal{L}\left(\sigma^{\prime}\right)$ such that

$$
T^{\prime} \vdash \phi \leftrightarrow \psi
$$

Write $\phi$ as $\phi(x, y)$ with $x$ a tuple of variables ranging over sorts not in $\Sigma$ and $y$ a tuple of variables ranging over sorts in $\Sigma$. By our hypotheses on $\sigma$ and $\sigma^{\prime}$, up to equivalence over $T^{\prime}$, we have

$$
\psi(x, y)=\bigvee_{j=1}^{n} \theta_{j}(x, y) \wedge \vartheta_{j}(\vec{\alpha}(\vec{t}(x, y)))
$$

Where $\theta_{j} \in \mathcal{L}(\sigma)$ and $\vartheta_{j} \in \mathcal{L}(\sigma\lceil\Sigma)$ are quantifier-free formulas, $\vec{\alpha}$ is a tuple of $\sigma^{\prime}$-terms, and $\vec{t}$ is a tuple of $\mathcal{L}(\sigma)$-terms with range in $\Sigma$. By considering each formula $\phi \wedge \theta_{j}$ separately, it suffices to work in the case that $n=1$ so that we drop the subscripts from $\theta$ and $\vartheta$.

If $\theta(x, y) \wedge \vartheta(\vec{\alpha}(\vec{t}(x, y)))$ is not equivalent (modulo $T \cup T_{q f-\Sigma, \sigma}$ ) to a quantifierfree $\mathcal{L}\left(\sigma \cup \sigma_{q f-\Sigma, \sigma}\right)$-formula, then it is consistent with $T$ that there be $\left(x^{\prime}, y^{\prime}\right)$ with

and

$$
\operatorname{tp}_{\mathcal{L}(\sigma \mid \Sigma)}(\vec{t}(x, y))=\operatorname{tp}_{\mathcal{L}(\sigma \mid \Sigma)}\left(\vec{t}\left(x^{\prime}, y^{\prime}\right)\right)
$$

$$
\operatorname{qftp}_{\mathcal{L}(\sigma)}(x, y)=\operatorname{qftp}_{\mathcal{L}(\sigma)}\left(x^{\prime}, y^{\prime}\right)
$$

and

$$
\models \phi(x, y) \wedge \neg \phi\left(x^{\prime}, y^{\prime}\right)
$$

By stable embeddability of $\Sigma$ (relative to $T$ ),

$$
\operatorname{tp}_{\mathcal{L}(\sigma \mid \Sigma)}(\vec{t}(x, y)) \vdash \operatorname{tp}_{\mathcal{L}(\sigma)}(\vec{t}(x, y))
$$

Thus, we can find such $x, y, x^{\prime}, y^{\prime}$ in some model $M \models T$ (which we may presume to be a reduct to $\mathcal{L}(\sigma)$ of a model of $\left.T^{\prime}\right)$ and a $\mathcal{L}(\sigma)$-automorphism $\tau: M \rightarrow M$ with $\tau(\vec{t}(x, y))=\left(\vec{t}\left(x^{\prime}, y^{\prime}\right)\right)$. If we suppose $M=\phi(x, y)$, then as $(x, y),\left(x^{\prime}, y^{\prime}\right)$, and $(\tau(x), \tau(y))$ all have the same $\mathcal{L}(\sigma)$-quantifier free type, we have $M \models \theta(x, y) \wedge$ $\theta\left(x^{\prime}, y^{\prime}\right) \wedge \theta(\tau(x), \tau(y))$. We have then

$$
\begin{aligned}
\phi(x, y) & \Rightarrow \phi(\tau(x), \tau(y)) \\
& \Rightarrow \theta(\tau(x), \tau(y)) \wedge \vartheta(\vec{\alpha}(\tau(\vec{t}(x, y))) \\
& \Rightarrow \theta\left(x^{\prime}, y^{\prime}\right) \wedge \vartheta\left(\vec{\alpha}\left(\vec{t}\left(x^{\prime}, y^{\prime}\right)\right)\right) \\
& \Rightarrow \phi\left(x^{\prime}, y^{\prime}\right)
\end{aligned}
$$

We return now to the case of valued $D$-fields. 
Lemma 7.3 If $K$ is a valued difference field with $v\left(\operatorname{Fix}(\sigma)^{\times}\right)=\Gamma=v K$ and $\tau: \bigcup_{n \in \omega} K_{n} \rightarrow \bigcup_{n \in \omega} K_{n}$ is an automorphism of the reduct to $\left\{K_{n}: n \in \omega\right\}$, then there is an elementary extension $\left(K^{*}, \tau^{*}\right)$ of $(K, \tau)$ which admits the structure of a valued difference field with angular components with respect to which $\tau^{*}$ is an automorphism.

Proof Let $\left(K^{*}, \tau^{*}\right) \succeq(K, \tau)$ be any uncountable saturated elementary extension.

We will define a homomorphism $\chi: \Gamma_{K^{*}} \rightarrow \lim _{n \in \omega}\left\{x \in K_{n}^{*}: \sigma_{n}(x)=x, x \neq\right.$ $0\}=: \mathcal{K}$ satisfying $\chi \circ v=\mathrm{id}$ and $\chi \circ \tau^{*}=\tau^{*} \circ \chi$. If we define $\mathrm{ac}_{n}: K_{n}^{*} \rightarrow k^{K^{*}}$ by $\operatorname{ac}_{n}(x):=\rho_{n}\left(\frac{x}{\chi(v(x))}\right)$, then we have the desired angular components.

We define $\chi$ by transfinite recursion ensuring that at each stage the domain of $\chi$ is a pure $\mathbb{Z}\left[\tau^{*}\right]$-submodule of $\Gamma_{K}$ of strictly smaller rank. That is, we express $\Gamma_{K^{*}}$ as an increasing union $\bigcup_{\alpha<\left|\Gamma_{K^{*}}\right|} \Gamma_{\alpha}$ of pure sub $\mathbb{Z}[\tau]$-submodules with $\operatorname{dim}_{\mathbb{Q}\left(\tau^{*}\right)} \mathbb{Q}\left(\tau^{*}\right) \otimes_{\mathbb{Z}\left[\tau^{*}\right]} \Gamma_{\alpha} / \lim _{\beta<\alpha} \Gamma_{\beta}=1$ and build an increasing sequence $\left\{\chi_{\alpha}\right\}$ of sections with $\operatorname{dom}\left(\chi_{\alpha}\right)=\Gamma_{\alpha}$. At stage $\alpha$, we have $\chi \uparrow \bigcup_{\beta<\alpha} \Gamma_{\beta}$ already defined and we try to extend the definition to all of $\Gamma_{\alpha}$.

Consider the partial type $\Pi:=\left\{c_{\gamma} \cdot c_{\delta}=c_{\gamma+\delta}: \gamma, \delta \in \Gamma_{\alpha}\right\} \cup\left\{c_{\gamma}=\chi(\gamma): \gamma \in \Gamma_{\beta}\right.$ for some $\beta<\alpha\} \cup\left\{\sigma\left(c_{\gamma}\right)=c_{\gamma}: \gamma \in \Gamma_{\alpha}\right\} \cup\left\{\tau\left(c_{\gamma}\right)=c_{\tau(\gamma)}: \gamma \in \Gamma_{\alpha}\right\}$. We claim that $\Pi$ is finitely satisfiable. Any finite subset of $\Pi$ is implied by by a formula of the form $\sigma\left(c_{\gamma}\right)=c_{\gamma} \wedge c_{\gamma}^{a} \cdot \chi(\delta)^{b}=c_{a \gamma+b \delta}$ for some $\gamma \in \Gamma_{\alpha}, \delta \in \lim _{\beta<\alpha} \Gamma_{\beta}$, and $a, b \in \mathbb{Z}\left[\tau^{*}\right]$. (Here we allow $\mathbb{Z}[\tau]$ to act on $(\mathcal{K}, \cdot)$ via $\left(\sum n_{i}\left(\tau^{*}\right)^{i}\right) \cdot x:=\prod\left(\tau^{*}\right)^{i}(x)^{n_{i}}$.) We can realize this formula by taking for $c_{\gamma}$ any element of $\mathcal{K}$ fixed by $\sigma$ with $v\left(c_{\gamma}\right)=\gamma$ and then defining $c_{a \gamma+b \delta}=\chi(b \delta) \cdot c_{\gamma}^{b}$. Thus, $\Pi$ is realizable in $\mathcal{K}$. Define $\chi(\gamma)=c_{\gamma}$.

Proposition 7.4 Theorem 6.3 implies Theorem 6.4.

Proof We prove first the resplendent relative completeness part of Theorem 6.4.

Let $\mathcal{L}^{\prime} \supseteq \mathcal{L}_{v d f}$ be some expansion of the language of valued difference fields by relations on $\left\{K_{n}: n \in \omega\right\}$.

Let $K$ and $L$ be $\sigma$-henselian fields of characteristic zero considered as an $\mathcal{L}^{\prime}$ structures. We assume that $\operatorname{Th}_{\mathcal{L}^{\prime}\left\lceil\left\{K_{n}: n \in \omega\right\}\right.}(K)=\operatorname{Th}_{\mathcal{L}^{\prime} \uparrow\left\{K_{n}: n \in \omega\right\}}(L)$ and that $K$ and $L$ have the same $\mathcal{L}^{\prime}$-atomic diagrams.

Passing to elementary extensions, we may assume that $K\left\lceil\left\{K_{n}: n \in \omega\right\} \cong_{\mathcal{L}^{\prime} \uparrow\left\{K_{n}: n \in \omega\right\}}\right.$ $L \uparrow\left\{K_{n}: n \in \omega\right\}$. By Lemma 7.3 applied to $\tau=$ id there are elementary extensions $K^{*} \succeq K$ and $L^{*} \succeq L$ which admit expansions to $\mathcal{L}^{\prime}\left(\left\{\mathbf{a c}_{n}: n \in \omega\right\}\right)$. Using the above assumption, we may assume that $\operatorname{Th}_{\mathcal{L}^{\prime}\left(\left\{a x_{n}: n \in \omega\right\}\right)}\left(K^{*}\left\lceil\left\{K_{n}: n \in\right.\right.\right.$ $\omega\})=\operatorname{Th}_{\mathcal{L}^{\prime}\left(\left\{\boldsymbol{x}_{n}: n \in \omega\right\}\right)}\left(L^{*}\left\lceil\left\{K_{n}: n \in \omega\right\}\right)\right.$ and that $K^{*}$ and $L^{*}$ have the same $\mathcal{L}^{\prime}\left(\left\{\mathrm{ac}_{n}: n \in \omega\right\}\right)$-atomic diagrams. By the resplendent completeness part of Theorem $6.3, K^{*} \equiv_{\mathcal{L}^{\prime}\left(\left\{\boldsymbol{x}_{n}: n \in \omega\right\}\right)} L^{*}$. Thus, $K \equiv_{\mathcal{L}} L$. As $\mathcal{L}^{\prime}, K$ and $L$ were arbitrary, we have shown that the theory of $D$-henselian fields of characteristic zero given together with a consistent atomic diagram in $\mathcal{L}_{\mathrm{vdf}}$ is resplendently complete relative to the leading terms.

We prove now the resplendent relative quantifier elimination component of the theorem. Fix again some expansion $\mathcal{L}^{\prime} \supseteq \mathcal{L}$ by predicates on the leading terms and $K$ some $\sigma$-henselian field of characteristic zero considered as an $\mathcal{L}^{\prime}$-structure.

We claim that $\left\{K_{n}: n \in \omega\right\}$ is stably embedded. Let $K$ be as in the previous paragraph and let $\tau$ be any automorphism of $K \backslash\left\{K_{n}: n \in \omega\right\}$. By Lemma 7.3 there is a $\sigma$-henselian field $K^{*}$ with angular components considered 
as an $\mathcal{L}^{\prime}\left(\left\{\mathrm{ac}_{n}: n \in \omega\right\}\right)$-structure such that $K \preceq\left(K^{*} \uparrow \mathcal{L}^{\prime}\right)$ and there is some $\tau^{*} \in \operatorname{Aut}_{\mathcal{L}^{\prime}\left(\left\{\mathbf{a}_{n}: n \in \omega\right\}\right)}\left(K^{*} \uparrow\left\{K_{n}: n \in \omega\right\}\right)$ with $\tau^{*} \supseteq \tau$. By Theorem 6.3 and Lemma 7.1 , there is an elementary extension $L \succeq K^{*}$ on which $\tau^{*}$ extends to an automorphism of all of $L$. This automorphism is, of course, also an automorphism of $L \uparrow \mathcal{L}$. Thus, every $\mathcal{L}^{\prime}$-automorphism of $K \uparrow\left\{K_{n}: n \in \omega\right\}$ extends to some $\mathcal{L}^{\prime}$-automorphism of some elementary extension of $K$ so that $\left\{K_{n}: n \in \omega\right\}$ is stably embdedded.

The main result now follows from Lemma 7.2.

\section{Tests for completeness and quantifier elimination}

Before we delve into the details of this chapter some words for mathematicians with allergies to set theory are in order. Our main algebraic problem concerns the extendibility of certain isomorphisms of valued $D$-fields. Set theory obstructs the solution of this problem in two ways. First, because there are uncountably many subsets of the residue field, it may happen that some instance of resplendent completeness and resplendent quantifier elimination concerns uncountable languages. If we wish to deal with these directly, then we must work with valued $D$-fields having cardinality well beyond that of the continuum and we may need to make rather strong set theoretic hypotheses. However, while the algebraic problem may push us into set theoretically perilous territory, the logical problems (Is a particular theory complete? Does it eliminate quantifiers?) concern formulas, finite strings of symbols, and can be reduced in a routine manner to the case of countable languages.

One (rather crude) interpretation of the work of Gödel and Cohen on the Continuum Hypothesis is that as far as ordinary mathematics is concerned it does not matter whether $2^{\aleph_{0}}=\aleph_{1}$ or not. While I do not subscribe to this view in general, it is valid for the problems considered in this paper. In our extension of isomorphism argument, we will need to express some valued $D$-field having the cardinality of the continuum as a direct limit of a chain of countable subfields. Unfortunately, this is only possible if the continuum hypothesis holds. However, the ultimate conclusions we which to reach (about completeness and elimination of quantifiers) are absolute. That is, if they are true when the continuum hypothesis holds, then they are also true when the continuum hypothesis fails. So, we can assume the continuum hypothesis when it is convenient and then assert our conclusion unconditionally.

A more detailed discussion of these completeness and quantifier elimination tests can be found in [9].

Recall that if $T$ is a complete theory in a first-order language $\mathcal{L}$ and $\kappa \geq|\mathcal{L}|$ is a cardinal, then $T$ has at most one saturated model of cardinality $\kappa$. Of course, if $\mathcal{M}$ and $\mathcal{N}$ are two isomorphic $\mathcal{L}$-structures, then they have the same theory. These observations give a test for completeness of a theory.

Test 8.1 Let $T$ be a theory in a first-order language $\mathcal{L}$. Suppose that $T$ has no finite models and that each consistent completion of $T$ has saturated models in each cardinality $\kappa>|\mathcal{L}|$. Then $T$ is complete if and only for any two saturated models of $T$ of the same cardinality are isomorphic.

Test 8.1 includes the extraneous hypothesis that the completions of $T$ have saturated models. In general, one needs to know $T$ very well or make some set theoretic hypotheses beyond the usual ZFC axioms (for example, the generalized continuum hypothesis (GCH) suffices) in order to verify this hypothesis. However, 
it is possible to make these additional hypotheses simply for the purpose of the test and then conclude unconditionally that $T$ is complete. That is, the assertion that a given theory in a countable language is complete is absolute (does not depend on the model of set theory) and the completeness of an arbitrary theory is equivalent to the completeness of its (or really, the set of its consequences) restrictions to countable sublanguages. We note this reduction to countable languages.

Proposition 8.2 A theory $T$ in a language $\mathcal{L}$ is complete if and only if for any countable sublanguage $\mathcal{L}^{\prime} \subseteq \mathcal{L}$ the restriction of $T$ to $\mathcal{L}^{\prime}$ is complete. Moreover, $T$ eliminates quantifiers in $\mathcal{L}$ if and only if for each countable sublanguage $\mathcal{L}^{\prime} \subseteq \mathcal{L}$ there is another countable language $\mathcal{L}^{\prime \prime}$ with $\mathcal{L}^{\prime} \subseteq \mathcal{L}^{\prime \prime} \subseteq \mathcal{L}$ and the restriction of $T$ to $\mathcal{L}^{\prime \prime}$ eliminates quantifiers.

With this reduction in place, it suffices to consider only countable languages.

Test 8.3 Let $T$ be a theory in a countable first-order language $\mathcal{L}$. Suppose that $T$ has no finite models. Then the following are equivalent.

- $T$ is complete.

- In any model of set theory in which the continuum hypothesis holds holds, if $\mathcal{M}=T$ and $\mathcal{N} \models T$ are saturated and have the same uncountable cardinality, then $\mathcal{M} \cong \mathcal{N}$.

- In some model of set theory in which the continuum hypothesis holds and there is some uncountable cardinal $\kappa$ such that any two saturated models of $T$ of cardinality $\kappa$ are isomorphic.

We have similar tests for quantifier elimination. As with completeness, the property of a theory admitting quantifier elimination is absolute. Thus, we can work in model of set theory in which saturated models exist abundantly. In such a universe, quantifier elimination for a complete theory is equivalent to condition that every partial automorphism (with a small domain) of a saturated model extends to an automorphism. Using a back-and-forth, one finds a more constructive version of this latter condition in terms of extending a partial automorphism to one new element.

Test 8.4 Let $T$ be a complete theory in a countable first-order language $\mathcal{L}$. The following are equivalent.

- $T$ eliminates quantifiers: For any formula $\varphi\left(x_{1}, \ldots, x_{n}\right) \in \mathcal{L}\left(x_{1}, \ldots, x_{n}\right)$ there is a quantifier-free formula $\vartheta\left(x_{1}, \ldots, x_{n}\right) \in \mathcal{L}\left(x_{1}, \ldots, x_{n}\right)$ such that $T \vdash\left(\forall x_{1}, \ldots, x_{n}\right) \varphi\left(x_{1}, \ldots, x_{n}\right) \leftrightarrow \vartheta\left(x_{1}, \ldots, x_{n}\right)$.

- In any model of set theory in which the continuum hypothesis holds, if $\mathcal{M}=$ $T$ is a saturated model and $f: A \rightarrow B$ is an $\mathcal{L}$-isomorphism where $A, B \subseteq \mathcal{M}$ are countable substructures, then there is an automorphism $\sigma: \mathcal{M} \rightarrow \mathcal{M}$ such that $\left.\sigma\right|_{A}=f$.

- If $\mathcal{M} \models T$ is an $\aleph_{1}$-saturated model, $A, B \subseteq \mathcal{M}$ are countable substructures, $f: A \rightarrow B$ is an $\mathcal{L}$-isomorphism, and $a \in \mathcal{M}$, then there is an extension of $f$ to an $\mathcal{L}$-embedding of the structure generated by a over $A$ into $\mathcal{M}$.

Since the tests for quantifier elimination and for completeness are so similar, we can combine them into a single extension of partial isomorphism test.

Test 8.5 Let $T$ be a theory in a countable first-order language $\mathcal{L}$. Suppose that $T$ has no finite models, that $T$ is complete with respect to the atomic theory (that 
is, for each atomic sentence $\psi$ of $\mathcal{L}$ either $T \vdash \psi$ or $T \vdash \neg \psi)$, and that $\mathcal{L}$ has at least one constant symbol. Then the following are equivalent.

- $T$ is complete and eliminates quantifiers.

- In any model of set theory in which the continuum hypothesis holds, if $\mathcal{M}, \mathcal{N}=T$ are saturated models of $T$ of the same uncountable cardinality, $A \subseteq \mathcal{M}$ and $B \subseteq \mathcal{N}$ are countable substructures of $\mathcal{M}$, and $f: A \rightarrow B$ is an $\mathcal{L}$-isomorphism, then there is an $\mathcal{L}$-isomorphism $g: \mathcal{M} \rightarrow \mathcal{N}$ such that $\left.g\right|_{A}=f$.

- If $\mathcal{M}, \mathcal{N} \models T$ are $\aleph_{1}$-saturated, $A \subseteq \mathcal{M}$ is a countable substructure, $f$ : $A \hookrightarrow \mathcal{N}$ is an $\mathcal{L}$-embedding, and $a \in \mathcal{M}$, then there is a substructure $A^{\prime}$ of $\mathcal{M}$ containing $A$ and $a$ and an extension of $f$ to an $\mathcal{L}$-embedding $g: A^{\prime} \hookrightarrow \mathcal{L}$.

It is this test that we use to prove Theorem 6.3. We take for $\mathcal{L}$ some expansion of $\mathcal{L}_{\mathrm{vdf}}^{\mathrm{ac}}$ by predicates on the leading terms. We take for $T$ the theory of $D$-henselian fields of characteristic zero together with a consistent atomic diagram and a complete theory in the restriction of $\mathcal{L}$ to the leading terms. It is worth noting that while in the definition of resplendent relative completeness and resplendent relative quantifier elimination arbitrary expansions of the base language are permitted, it suffices to consider only countable languages.

\section{Reduction to Equicharacteristic Zero}

We have stated on many occasions that the theory of the relative Frobenius (the unique lifting of the Frobenius automorphism to an automorphism of the Witt vectors) motivates our study of more general $D$-henselian fields. In this section we show that the relative Frobenius fits into the framework of $D$-henselian fields and that standard coarsening arguments permit us to reduce our general problem to the case of pure characteristic zero $D$-henselian fields.

We start with some observations about the relation between linear $D$-closedness, linear difference closedness, and $D$-henselianness.

Lemma 9.1 Let $(K, \sigma)$ be a difference field. Let $e \in K^{\times}$. Give $K$ the structure of a $D$-field by setting $D(x):=\frac{\sigma(x)-x}{e}$. Then, $K$ is linearly $D$-closed if and only

if $K$ is linearly difference closed (ie, for any nonzero polynomial $\sum_{j=0}^{m} a_{j} X^{j} \in K[X]$ the linear difference operator $\sum_{j=0}^{m} a_{j} \sigma^{j}$ is surjective on $K$ ).

\section{Proof:}

An easy induction shows for any natural number $n$ that $D^{n}=\left(\prod_{j=0}^{n-1} \sigma^{j}(e)\right) \sigma^{n}+$ \{lower order terms $\}$ and $\sigma^{n}=\left(\prod_{j=0}^{n-1} \sigma^{j}(e)^{-1}\right) D^{n}+\{$ lower order terms $\}$. Thus, for any sequence $\left(a_{0}, \ldots, a_{m}\right) \in K^{m+1}$ we have

$$
\begin{aligned}
\sum_{j=0}^{m} a_{j} D^{j} & =a_{m}\left(\prod_{j=0}^{m-1} \sigma^{j}(e)\right) \sigma^{m}+\text { lower order terms } \\
\sum_{j=0}^{m} a_{j} \sigma^{j} & =a_{m}\left(\prod_{j=0}^{m-1} \sigma^{j}(e)^{-1}\right) D^{m}+\text { lower order terms }
\end{aligned}
$$


As $a_{m} \neq 0$, then the operator $\sum_{j=0}^{m} a_{j} D^{j}$ is equal to a difference operator having a non-zero leading co-efficient. As $K$ is linearly difference closed, this operator is therefore surjective. Likewise, linear $D$-closedness implies linear difference closedness.

Corollary 9.2 If $K$ is a field of characteristic $p>0$ with no extensions of degree $p, e \in K^{\times}, n \in \mathbb{Z} \backslash\{0\}$ and $D: K \rightarrow K$ is defined by $D(x):=\frac{x^{p^{n}}-x}{e}$, then $K$ is linearly D-closed.

Lemma 9.3 If $(K, v, D)$ is D-henselian, then $K$ is linearly D-closed.

Proof: Let $L=\sum_{j=0}^{n} a_{j} D^{j} \in K[D]$ be a non-zero linear $D$-operator. Let $\alpha \in K$. If $\alpha=0$, then $L(0)=0=\alpha$ so that we may assume that $\alpha \neq 0$. Let $\epsilon \in K$ with $D \epsilon=0$ and $v(\epsilon)=\max \left\{v(\alpha)-v\left(a_{j}\right)\right\}$. Let $Q(Y):=\sum_{j=0}^{n} \frac{a_{j} \epsilon}{\alpha} D^{j}(Y)-1$. By our choice of $\epsilon, Q(Y) \in \mathcal{O}_{K}\langle X\rangle_{D}$ and the reduction of $Q, \pi(Q)$, is a non-constant affine $D$-polynomial over the residue field of $K$. Since the residue field of $K$ is linearly $D$-closed, we can find some $b \in \mathcal{O}_{K}$ such that $\pi(Q(b))=0$. As $\pi(Q)$ is residually affine, $v\left(\frac{\partial}{\partial X_{i}} Q(b)\right)=0$ for some $i$. Thus, DHL applies to $Q$ at $b$ and we can find some $a \in \mathcal{O}_{K}$ with $v(a-b)>0$ and $Q(a)=0$. Let $\tilde{a}:=a \epsilon$. Then $L(\tilde{a})=\alpha(Q(a)+1)=\alpha$. Thus, $L$ is surjective on $K$.

As with pure fields, $D$-Hensel's lemma takes many forms. In the next lemma we show the equivalence between the version of $D$-Hensel's lemma already given and an ostensibly stronger version in which the approximate solution is not assumed to be a simple solution in the residue field.

Lemma 9.4 Let $(K, v, D)$ be a valued D-field with enough constants and linearly D-closed residue field. The following are equivalent.

1. For all D-polynomials $P(X) \in \mathcal{O}_{K}\langle X\rangle$ and elements $a \in \mathcal{O}_{K}$ such that $v(P(a))>0=\min \left\{v\left(\frac{\partial}{\partial X_{i}} P(a)\right): i \in \omega\right\}$, there is some $b \in \mathcal{O}_{K}$ with $P(b)=0$ and $v(a-b)=v(P(a))$.

2. Given $P(X) \in \mathcal{O}_{K}\langle X\rangle$ and $a \in \mathcal{O}_{K}$ define $\gamma:=\min \left\{v\left(\frac{\partial}{\partial X_{i}} P(a)\right): i \in \omega\right\}$. If $v(P(a))>2 \gamma$, then there is some $b \in \mathcal{O}_{K}$ with $P(b)=0$ and $v(a-b) \geq$ $v(P(a))-\gamma$.

Proof: The implication from (2) to (1) is immediate so we concentrate on proving (1) to $(2)$.

Let $P(X) \in \mathcal{O}_{K}\langle X\rangle_{D}$ and $a \in \mathcal{O}_{K}$ be given with $\gamma=\min \left\{v\left(\frac{\partial}{\partial X_{i}} P(a)\right): i \in \omega\right\}$ having $v(P(a))>2 \gamma$.

If $P(a)=0$, then there is nothing to prove so we assume now that $P(a) \neq 0$.

Let $\epsilon \in K^{D}$ with $v(\epsilon)=v(P(a))-\gamma$. Let $Q(Y):=\frac{1}{P(a)} P(a+\epsilon Y)$. I claim that $Q(Y) \in \mathcal{O}_{K}\langle Y\rangle_{D}$. To see this, expand

$$
P(a+\epsilon Y) \equiv P(a)+\sum \frac{\partial}{\partial X_{i}} P(a) \epsilon D^{i} Y+\left(\epsilon^{2}\right)
$$

Of course, $P(a)$ divides $P(a)$, so the constant term of $Q(Y)$ is integral. By our choice of $\epsilon$ and the definition of $\gamma$, 


$$
\begin{aligned}
v\left(\frac{\partial}{\partial X_{i}} P(a) \epsilon\right) & =v\left(\frac{\partial}{\partial X_{i}} P(a)\right)+v(\epsilon) \\
& \geq v(P(a))
\end{aligned}
$$

Thus, the linear term of $Q(Y)$ is integral. Finally, we have

$$
\begin{aligned}
v\left(\epsilon^{2}\right) & =2 v(P(a))-2 \gamma \\
& =v(P(a))+(v(P(a))-2 \gamma) \\
& >v(P(a))
\end{aligned}
$$

which implies that the higher terms in $Q(Y)$ have co-efficients in the maximal ideal of $\mathcal{O}_{K}$.

So, not only is $Q(Y)$ integral, but its reduction is a nonzero affine $D$-polynomial. As the residue field is linearly $D$-closed, there is some $b \in K$ such that $v(Q(b))>0$ and $v(b) \geq 0$. By DHL in the original form, there is some $c \in \mathcal{O}_{K}$ such that $Q(c)=0$ and $v(c-b)=v(Q(b))$. Set $d:=a+\epsilon c$. Then $P(d)=0$ and $v(a-d)=$ $v(\epsilon)+v(c)=v(\epsilon)=v(P(a))-\gamma$.

Lemma 9.5 If $(K, v, D)$ is a D-henselian field and $w$ is a coarsening of the valuation $v$, then $(K, w, D)$ is also a D-henselian field.

Proof: We check the axioms.

Let $\tilde{w}: v K \rightarrow w K$ be the homomorphism for which $w=\tilde{w} \circ v$. Note that $\tilde{w}$ is order preserving.

Axiom 1 makes no mention of the valuation so it remains true.

For Axiom 2 let $x \in K$, then by hypothesis $v D x \geq v x$ and $v \sigma(x)=v x$. Applying $\tilde{w}$, we obtain $w(D x)=\tilde{w} \circ v D x \geq \tilde{w} \circ v x=w x$ and $w(\sigma(x))=\tilde{w} \circ$ $v(\sigma(x))=\tilde{w} \circ v x=w x$.

The main parts of Axioms 3 and 4 state merely that the extra sorts are interpreted as the value group and residue field. This does not change upon passage from $v$ to $w$. The assertion in Axiom 3 that $\pi$ is a map of $\mathcal{L}_{D \text {-ring }}\left(\sigma, \sigma^{-1}\right)$-structures follows from Axiom 2 once one knows thst $\pi$ actually is the reduction modulo $\mathfrak{m}_{K, w}$ map.

For Axiom 5 let $\gamma \in w K$. Let $\tilde{\gamma} \in v K$ with $\tilde{w}(\tilde{\gamma})=\gamma$. By Axiom 5 for $v$ we can find $\epsilon \in K$ with $v \epsilon=\tilde{\gamma}$ and $D \epsilon=0$. Apply $\tilde{w}$ and we see that $w \epsilon=\tilde{w} \circ v(\epsilon)=$ $\tilde{w}(\tilde{\gamma})=\gamma$.

The only axiom requiring real proof is Axiom 6, $D$-Hensel's lemma. For this we use the strengthened version of $D$-Hensel's lemma. If $P(X) \in \mathcal{O}_{K, w}\langle X\rangle$ and $a \in \mathcal{O}_{K, w}$ with $w(P(a))>0=w\left(\frac{\partial}{\partial X_{i}} P(a)\right)$ for some $i$, we can scale so that $P(X) \in \mathcal{O}_{K, v}$ and $a \in \mathcal{O}_{K, v}$. (Note: this move uses the fact that $(K, v, D)$ has enough constants.) The hypothesis that $w\left(\frac{\partial}{\partial X_{i}} P(a)\right)=0$, does not mean that $v\left(\frac{\partial}{\partial X_{i}} P(a)\right)=0$. Rather, because $w$ is a refinement of $v$, we can conclude from this and $w(P(a))>0$ that $v(P(a))>2 v\left(\frac{\partial}{\partial X_{i}} P(a)\right)$. By the strengthened version of DHL, there is some $b$ such that $P(b)=0$ and $v(a-b)=v(P(a))-v\left(\frac{\partial}{\partial X_{i}} P(a)\right)$. We have $w(a-b)=\tilde{w} \circ v(a-b)=\tilde{w} \circ v(P(a))-\tilde{w} \circ v\left(\frac{\partial}{\partial X_{i}} P(a)\right)=w(P(a))$ as required. 
With the above lemmata in place, we can reduce our main problem to the case of pure characteristic zero valued $D$-fields.

Proposition 9.6 Theorem 6.3 is equivalent to the corresponding statement restricted to D-henselian of residue characteristic zero.

Proof The restriction to residue characteristic zero is a special case of Theorem 6.3 so that the left to right implication is immediate. We concentrate on proving the right to left implication.

Using Test 8.5, the hypothesis of this proposition takes the form:

Hypothesis 9.7 In any model of set theory in which the continuum hypothesis holds, if

- $\mathcal{L} \supseteq \mathcal{L}_{\mathrm{vdf}}^{\mathrm{ac}}$ is an expansion of the language $\mathcal{L}_{\mathrm{vdf}}^{\mathrm{ac}}$ by at most countably many predicates on the leading terms,

- $K$ and $L$ are saturated D-henselian fields of equicharacteristic zero of the same cardinality considered as $\mathcal{L}$-structures,

- $\Gamma_{K} \equiv_{\mathcal{L}} \Gamma_{L}$,

- $\bigcup R_{n}(K) \equiv_{\mathcal{L}} \cup R_{n}(L)$

- $\Gamma_{K}$ and $\bigcup R_{n}(K)$ are $\mathcal{L}$-quantifier eliminable, and

- $f: A \rightarrow B$ is an isomorphism between countable substructures of $K$ and $L$,

then $f$ extends to an isomorphism between $K$ and $L$.

Using the same test, the conclusion takes the form.

\section{Conclusion 9.8 If}

- $\mathcal{L} \supseteq \mathcal{L}_{\mathrm{vdf}}^{\mathrm{ac}}$ is a countable expansion of $\mathcal{L}_{\mathrm{vdf}}^{\mathrm{ac}}$ by predicates on the leading terms,

- $K$ and $L$ are $\aleph_{1}$-saturated $D$-henselian fields of characteristic zero with $\mathcal{L}$ elementarily equivalent and quantifier eliminable value groups and residue fields,

- $A \subset K$ is a countable substructure,

- $a \in K$ is an element, and

- $f: A \hookrightarrow L$ is an $\mathcal{L}$-embedding,

then $f$ extends to an embedding of $A(\langle a\rangle)$ into an elementary extension of $L$.

So we may assume the continuum hypothesis and take two saturated mixed characteristic valued $D$-field $K$ and $L$ with the cardinality of the continuum satisfying the above hypotheses for some language $\mathcal{L}$. Furthermore, We may (and do) assume that the restriction of $f$ to $\bigcup_{n \in \omega} R_{n}(K)$ is an $\mathcal{L}$-isomorphism between $\bigcup_{n \in \omega} R_{n}(K)$ and $\bigcup_{n \in \omega} R_{n}(L)$ and the restriction of $f$ to $\Gamma_{K}$ is an $\mathcal{L}$-isomorphism between $\Gamma_{K}$ and $\Gamma_{L}$.

Let $w_{K}$ be the valuation on $K$ having valuation ring $\mathcal{O}_{K}\left[\frac{1}{p}\right]$ and $w_{L}$ the valuation on $L$ with valuation ring $\mathcal{O}_{L}\left[\frac{1}{p}\right]$. The structures $\left(K, w_{K}, D\right)$ and $\left(L, w_{L}, D\right)$ are valued $D$-fields of pure characteristic zero. Our task is to show that the hypotheses of Theorem 6.3 are true of these structures and that an embedding with respect to an expansion of $\mathcal{L}_{\mathrm{vdf}}^{\mathrm{ac}}$ by predicates on $w$-zero leading terms induces an embedding of $K$ into $L$ for $\mathcal{L}_{\mathrm{vdf}}^{\mathrm{ac}}$.

The saturation hypotheses on $L$ and $K$ imply that we may recover the $w_{K^{-}}$ residue field of $K$ (respectively, the $w_{L}$-residue field of $L$ ) from the residue rings $R_{n}(K)$ and $R_{n}(L)$. That is, $\aleph_{1}$-saturation implies that the natural maps 


$$
\mathcal{O}_{K, v} / \mathfrak{m}_{K, w_{K}} \stackrel{\psi_{K}}{\longrightarrow} \lim _{n \leftrightarrows \infty} R_{n}(K)
$$

and

$$
\mathcal{O}_{L, v} / \mathfrak{m}_{L, w_{L}} \stackrel{\psi_{L}}{\longrightarrow} \lim _{n \rightarrow \infty} R_{n}(L)
$$

are isomorphisms of multiplicative monoids. These maps preserve more than just the multiplicative structure. They are isomorphisms of $\mathcal{L}_{D \text {-ring }}\left(\sigma, \sigma^{-1}\right)$-structures as well: if $x, y \in K$ and $w_{K}(x+y)=w_{K}(x)=w_{K}(y)$, then for some $n \in \omega$ we have $v(x+y)<\min \{v x+n v(p), v y+n v(p)\}$ so that for $m>n$ the expression $\pi_{2 m}(x)+{ }_{2 m, m} \pi_{2 m}(y)=\pi_{m}(x+y)$ is well-defined. Likewise, the $D$ and $\sigma$-structure on $K_{\mathfrak{m}_{K, w_{K}}}$ is determined by the structure on $\left\{K_{n}\right\}_{n \in \omega}$. Thus, the map $f$ induces an isomorphism between the zero-leading term structure of $K$ (with respect to $w_{K}$ ) and the zero-leading term structure of $L$ (with respect to $w_{L}$ ).

We need to work to produce an angular component function for the coarsened valuation as the coarsened $\mathcal{L}_{\mathrm{vdf}}^{\mathrm{ac}}$ structure is not canonically determined by the fine $\mathcal{L}_{\mathrm{vdf}}^{\mathrm{ac}}$ structure. However, the indeterminacy may be traced to the choice of a section of $K^{\times} / \mathcal{O}_{K, v}^{\times} \rightarrow K^{\times} / \mathcal{O}_{K, v}\left[\frac{1}{p}\right]^{\times}$so that we can keep it under control.

We note that $\mathfrak{m}_{K, w}=\bigcap_{n \in \omega} p^{n} \mathcal{O}_{K, v}$. Thus, the angular component functions

$$
K^{\times} \stackrel{\mathrm{ac}_{n}}{\longrightarrow}\left(\mathcal{O}_{K} / p^{n+} \mathcal{O}_{K}\right)^{\times}
$$

patch together to give a section

$$
K^{\times} / 1+\mathfrak{m}_{K, w} \stackrel{\alpha}{\longrightarrow}\left(\mathcal{O}_{K} / p^{\infty} \mathcal{O}_{K}\right)^{\times}
$$

of the inclusion $\left(\mathcal{O}_{K} / \mathfrak{m}_{K, w}\right)^{\times} \hookrightarrow K^{\times} / 1+\mathfrak{m}_{K, w}$. However, $\alpha$ is not an angular component function. For $\alpha$ to be an angular component function we would need $\alpha$ restricted to $\mathcal{O}_{K}\left[\frac{1}{p}\right]^{\times} / 1+\mathfrak{m}_{K, w}$ to be the identity, but this is not the case as, for instance, $\alpha(p)=1$.

The coarsened valuation $w_{K}$ corresponds to the exact sequence

$$
\begin{aligned}
& 1 \rightarrow \mathcal{O}_{K}\left[\frac{1}{p}\right]^{\times} / \mathcal{O}_{K}^{\times} \rightarrow K^{\times} / \mathcal{O}_{K}^{\times} \rightarrow K^{\times} / \mathcal{O}_{K}\left[\frac{1}{p}\right]^{\times} \rightarrow 1 \\
& 1 \rightarrow \Gamma_{v / w} \rightarrow \Gamma_{v} \rightarrow \quad \begin{array}{lll}
\| & & \\
\Gamma_{w} & \rightarrow &
\end{array}
\end{aligned}
$$

Let $\vartheta: \Gamma_{v} \rightarrow \Gamma_{v / w}$ be a section of the inclusion $\Gamma_{v / w} \hookrightarrow \Gamma_{v}$.

From the splittings

$$
K^{\times} / 1+p^{n} \mathcal{O}_{K} \stackrel{\mathfrak{x}_{n}}{\longrightarrow}\left(\mathcal{O}_{K} / p^{n} \mathcal{O}_{K}\right)^{\times}
$$

we obtain splittings

$$
\Gamma_{v} \stackrel{\chi_{n}}{\longrightarrow} K^{\times} / 1+p^{n} \mathcal{O}_{K}
$$

of the sequences

$$
1 \longrightarrow\left(\mathcal{O}_{K} / p^{n} \mathcal{O}_{K}\right)^{\times} \longrightarrow K^{\times} / 1+p^{n} \mathcal{O}_{K} \stackrel{v}{\longrightarrow} \Gamma_{v} \longrightarrow 1
$$

defined by the relation $\mathrm{ac}_{n} \circ \chi_{n} \equiv 1$.

Let $\chi:=\lim _{n \rightarrow \infty} \chi_{n}: \Gamma_{v} \rightarrow \lim _{n \rightarrow \infty} K^{\times} / 1+p^{n} \mathcal{O}_{K}=K_{\mathfrak{m}_{K, w}}$. Let $\beta: K^{\times} / 1+\mathfrak{m}_{K, w} \rightarrow$ $\left(\mathcal{O}_{K}\left[\frac{1}{p}\right] / p^{\infty} \mathcal{O}_{K}^{n \rightarrow \infty}\left[\frac{1}{p}\right]\right)^{\times}$be defined by $\beta(x):=\alpha(x) \chi(\vartheta(v x))$. This function will serve

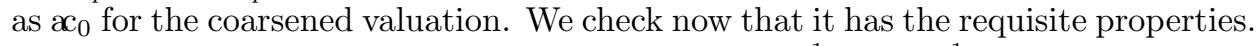

First, we remark that $\beta$ does take values in $\left(\mathcal{O}_{K}\left[\frac{1}{p}\right] / \mathfrak{m}_{K, w}\left[\frac{1}{p}\right]\right)^{\times}$as claimed. For any $x \in K$ we have $\vartheta(v x) \in \Gamma_{v / w}$ so that $\chi_{n}\left(\vartheta(v x) \in \mathcal{O}_{K}\left[\frac{1}{p}\right]^{\times} / 1+p^{n} \mathcal{O}_{K}\right.$. Thus, 


$$
\begin{aligned}
\chi\left(\vartheta(v x) \in \mathcal{O}_{K}\left[\frac{1}{p}\right]^{\times} / 1+\mathfrak{m}_{K, w}\right. & =\mathcal{O}_{K}\left[\frac{1}{p}\right]^{\times} / 1+\mathfrak{m}_{K, w}\left[\frac{1}{p}\right] \\
& =\left(\mathcal{O}_{K} / p^{\infty} \mathcal{O}_{K}\left[\frac{1}{p}\right]\right)^{\times}
\end{aligned}
$$

By construction, $\alpha(x) \in \mathcal{O}_{K}^{\times} / 1+\mathfrak{m}_{K, w} \hookrightarrow\left(\mathcal{O}_{K}\left[\frac{1}{p}\right] / \mathfrak{m}_{K, w}\left[\frac{1}{p}\right]\right)^{\times}$. Thus, $\beta(x) \in$ $\left(\mathcal{O}_{K, w} / \mathfrak{m}_{K, w}\right)^{\times}$.

Secondly, $\beta$ is a homomorphism. This is clear from the construction.

Thirdly, $\beta$ is a section. Let $x \in\left(\mathcal{O}_{K, w} / \mathfrak{m}_{K, w}\right)^{\times}$. Write $x=\tilde{x} \chi(v x)$. Note that $v(\tilde{x})=0$ so that $\tilde{x} \in \mathcal{O}_{K, v}^{\times} / 1+\mathfrak{m}_{K, w}$. We compute

$$
\begin{aligned}
\beta(x) & =\alpha(\tilde{x} \cdot \chi(v x)) \cdot \chi(v(\tilde{x} \cdot \chi(v x))) \\
& =\alpha(\tilde{x}) \cdot \alpha(\chi(v x)) \cdot \chi(v(\tilde{x})) \cdot \chi(v(\chi(v x))) \\
& =\tilde{x} \cdot 1 \cdot 1 \cdot \chi(v x) \\
& =x
\end{aligned}
$$

By Proposition 4.7 , the fact that $\beta$ is a section of $\left(\mathcal{O}_{K, w} / \mathfrak{m}_{K, w}\right)^{\times} \hookrightarrow K_{\mathfrak{m}_{K, w}}$ implies already that $\beta$ preserves addition as far as this makes sense.

Fourthly, $\beta$ preserves the difference structure.

$$
\begin{aligned}
\beta(\sigma(x)) & =\alpha(\sigma(x)) \cdot \chi(\vartheta(v \sigma(x))) \\
& =\lim _{n \leftrightarrows \infty} \operatorname{ac}_{n}(\sigma(x)) \cdot \lim _{n \leftrightarrows \infty} \chi_{n}(\vartheta(v x)) \\
& =\lim _{n \leftrightarrows \infty} \sigma\left(\operatorname{ac}_{n}(x)\right) \cdot \lim _{n \leftrightarrows \infty} \chi_{n}(\vartheta(v x)) \\
& =\sigma(\alpha(x)) \cdot \chi(\vartheta(v x)) \\
& =\sigma(\alpha(x) \cdot \chi(\vartheta(v x))) \\
& =\sigma(\beta(x))
\end{aligned}
$$

The penultimate equality uses the fact that the image of $\chi$ is contained in the fixed field of $\sigma$. Why is this? An element $x$ is equal to $\chi(\gamma)$ if and only if $v x=\gamma$ and $\alpha(x)=1$. The element $\sigma(x)$ satisfies the same defining conditions.

Finally, $\beta$ preserves the $D$-structure as far as this makes sense. The section $\chi$ also maps to $D$-constants as we have the functional equality $\alpha \circ \chi \equiv 1$. Thus, for any $\gamma$ we have $w(D(\chi(\gamma)))>w(\chi(\gamma))=\gamma$ for otherwise $\alpha(D(\chi(\gamma)))=D(\alpha(\chi(\gamma)))=$ $D(1)=0$ which is impossible.

So, let $x \in K^{\times} / 1+\mathfrak{m}_{K, w}$. Let $\tilde{x} \in K^{\times}$lift $x$. We suppose that $w D \tilde{x}=w \tilde{x}$. This means that $v D \tilde{x}=v \tilde{x}+\gamma<v \tilde{x}+n v(p)$ for some $n \in \mathbb{Z}_{+}$. Write $x=\bar{x} \chi(v x)$. Let $\tilde{\bar{x}} \in K^{\times}$lift $\bar{x}$. Then as $w D y>w y$ for any $y$ lifting $\chi(v x)$ we have $\pi_{0, w}(D \tilde{x})=$ 
$\pi_{0, w}(D \tilde{\bar{x}}) \cdot \chi(v x)$. We compute

$$
\begin{aligned}
\beta\left(\pi_{0, w}(D \tilde{x})\right) & =\beta\left(\pi_{0, w}((D \tilde{\bar{x}})) \chi(v x)\right) \\
& =\beta\left(\pi_{0, w}(D \tilde{\bar{x}})\right) \chi(\vartheta(v(\chi(v x))) \\
& =\alpha\left(\pi_{0, w}(D(\tilde{\bar{x}})) \chi(\vartheta(v D \tilde{\bar{x}})) \chi(\vartheta(v x))\right. \\
& =D \alpha(\bar{x}) \chi(\vartheta(\gamma)) \chi(\vartheta(v x)) \\
& =D \alpha(\bar{x}) \chi(\vartheta(\gamma+v x)) \\
& =D(\alpha(\bar{x}) \cdot \chi(\vartheta(v(D \tilde{x})))) \\
& =D(\beta(x))
\end{aligned}
$$

In order to meet the hypotheses of Theorem 6.3 , we replace $L$ by a $|K|^{+}$saturated elementary extension $M$ of $L$. (Note that $\left(L, w_{L}\right)$ is not even $\aleph_{1}$-saturated.) By Lemma 9.5, $\left(L, w_{L}, D\right)$ is $D$-henselian. So, assuming Theorem 6.3 just for equicharacteristic zero we obtain an embedding $g:\left(K, w_{K}, D\right) \rightarrow(M, w, D)$.

We use now the flexibility in the choice of $\mathcal{L}$. The $w$-residue field inherits a trace of the valuation $v$ on $K$. That is, we may (and do) continue to consider $k_{K, w}$ has having the valuation $v$. From this (and $w$ ) we fully recover $v$. Thus the embedding $K \rightarrow M$ can be taken to preserve $v$ as well!

\section{The proofs in equicharacteristic zero}

In the previous section we reduced the proof of the main theorem to the case of pure characteristic zero $D$-henselian fields. The proof we present in this section is a variant of the proof of the main theorem in [12]. While the statement The proof of the main theorem in [12] goes through with only minor changes. may be true, we present the details especially where the "minor changes" are not obvious.

Let us recall what needs to be proved. We are given two $\aleph_{1}$-saturated valued $D$ fields of pure characteristic zero $M_{1}$ and $M_{2}$ in some countable language $\mathcal{L}$ extending $\mathcal{L}_{\text {vdf }}^{\text {ac }}$ with some not necessarily new predicates on the sort $K_{0}$. We assume that $k_{M_{1}} \equiv k_{M_{2}}, \Gamma_{M_{1}} \equiv \Gamma_{M_{2}}$, and that the residue field and value group eliminate quantifiers in $\mathcal{L}$. We assume that $A \subseteq M_{1}$ is a countable substructure and that $f: A \hookrightarrow M_{2}$ is an $\mathcal{L}$-embedding and that $a \in M_{1}$. We need to show that $f$ extends to an embedding of $A(\langle a\rangle)$ into $M_{2}$.

Convention 10.1 For the remainder of this section, "valued $D$-field" means "valued $D$-field of equicharacteristic zero with an angular component function of level m."

Definition 10.2 If $P(X)=\sum p_{\alpha} \prod\left(D^{j} X\right)^{\alpha_{j}} \in K\langle X\rangle_{D}$ is a $D$-polynomial over the valued $D$-field $K$ and $x \in K$ is an element with $v x=\gamma \in \Gamma_{K}$, then one expects $v(P(x))=\min \left\{v\left(p_{\alpha}\right)+|\alpha| \gamma: \alpha \in \omega^{<\omega}\right\}$. We say that $P$ has the expected value at $x$ if $v P(x)$ is as expected.

The next lemma shows that if we control the valuation well enough, then we also control the angular component structure.

Lemma 10.3 If $K$ is a valued D-field, $P(X) \in K\langle X\rangle_{D}$ is a D-polynomial, $x \in K$ with $v x=\gamma \in \Gamma_{K}, v\left(D^{j} x\right)=\gamma$ for $j \leq \operatorname{ord} P$, and $P$ has the expected 
valuation at $x$, then

$$
\operatorname{ac}_{0}(P(x))=\sum_{\left\{\alpha: v p_{\alpha}+|\alpha| \gamma=v P(x)\right\}} \mathrm{ac}_{0}\left(p_{\alpha}\right) \prod_{j=0}^{\infty} D^{j} \mathrm{ac}_{0}(x)^{\alpha_{j}}
$$

Proof Our hypothesis is that $v D^{j} x=\gamma$ for $j \leq \operatorname{ord} P$ implies that

$$
v\left(p_{\alpha} \prod\left(D^{j} x\right)^{\alpha_{j}}\right)=v p_{\alpha}+|\alpha| \gamma
$$

for each multi-index $\alpha$. Let $T:=\left\{\alpha: v p_{\alpha}+|\alpha| \gamma=v P(x)\right\}$. By the ultrametric triangle inequality, we have

$$
v(P(x))=v\left(\sum_{\alpha \in T} p_{\alpha} \prod\left(D^{j} X\right)^{\alpha_{j}}\right)
$$

Thus, by Lemma 4.7 we have

$$
\begin{aligned}
\operatorname{ac}_{0}(P(x)) & =\sum_{\alpha \in T} \operatorname{ac}_{0}\left(p_{\alpha} \prod\left(D^{j} X\right)^{\alpha_{j}}\right) \\
& =\sum_{\alpha \in T} \operatorname{ac}_{0}\left(p_{\alpha}\right) \prod\left(D^{j}\left(\operatorname{ac}_{0}(x)\right)^{\alpha_{j}}\right.
\end{aligned}
$$

as claimed.

Lemma 10.4 Let $K$ be a valued $D$-field considered as an $\mathcal{L}$-structure with $L$ and $L^{\prime}$ two immediate extensions. If $L \cong{\widetilde{\mathcal{L}_{\mathrm{vdf} K}}} L^{\prime}$, then $L \cong_{\mathcal{L}_{K}} L^{\prime}$.

Proof Let $g: L \rightarrow L^{\prime}$ be an $\mathcal{L}_{\mathrm{vdf} K}$-isomorphism. Then $g$ induces the identity map on $L_{0}=K_{0}=L_{0}^{\prime}$. As the new functions and relations in $\mathcal{L}_{K}$ are all defined on the leading terms exclusively, the map $g$ respects them as well.

We need to pin down the structure on the henselization of a valued $D$-field in terms of the structure on the original field.

Lemma 10.5 If $K$ is a valued $D$-field considered as an $\mathcal{L}$-structure, then the henselization $K^{h}$ of $K$ (or more accurately, the field of fractions of the henselization, $\mathcal{O}_{K}^{h}$, of the ring of integers of $K$ ) has a unique structure of a valued $D$-field in the language $\mathcal{L}_{K}$.

Proof Lemma 7.11 of [12] shows that when $v(e)>0$, there is a unique $\mathcal{L}_{\mathrm{vdf}} K^{-}$ structure on $K^{h}$. In the case that $v(e)=0, D$ is interdefinable with $\sigma$ (and with $\left.\sigma^{-1}\right)$. By the universal property of the henselization $\sigma: \mathcal{O}_{K} \rightarrow \mathcal{O}_{K} \hookrightarrow \mathcal{O}_{K}^{h}$ induces a unique map $\sigma: \mathcal{O}_{K}^{h} \rightarrow \mathcal{O}_{K}^{h}$ as does $\sigma^{-1}$. Hence, in this case as well there is a

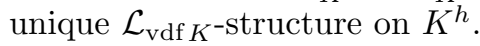

As a general rule $K_{0}=\left(K^{h}\right)_{0}$. Thus, by Lemma 10.4 the $\mathcal{L}_{K^{-}}$-structure on $K^{h}$ is also determined.

The next lemma concerns the structure of an extension obtained by adjoining a new element to the residue field. The proof of the corresponding lemma in [12] (Lemma 7.12) used the hypothesis that $v(e)>0$ substantially, though the use is removable. The proof given below recasts that proof with this extraneous hypothesis removed. 
Lemma 10.6 If $K$ is a valued $D$-field considered as an $\mathcal{L}$-structure, $p(x) \in$ $S_{1, k}\left(k_{K}\right)$ is a one-type in the residue field sort, $P(X) \in \mathcal{O}_{K}\langle X\rangle_{D}$ is a D-polynomial over $K$ for which

- $p(x) \vdash \pi(P)(x)=0$,

- $P$ and $\pi(P)$ have the same total degree, and

- if $Q(X) \in \mathcal{O}_{K} / \mathfrak{m}_{K}\langle X\rangle$ with $p(x) \vdash Q(x)=0$, then $\pi(P) \ll Q$;

then there is a unique $\mathcal{L}_{K}$-structure on $L=K(\langle b\rangle)$ for which $P(b)=0, v(b) \geq 0$, and $\pi(b) \models p$.

Proof Lemma 7.12 of [12] shows the existence and uniqueness of the $\mathcal{L}_{\mathrm{vdf} K^{-}}$ structure on $L$ in the case of $v e>0$. Moreover, every element $c$ of $L$ is of the form $\frac{R(b)}{Q(b)}$ with $R, Q \ll P$. It follows that $R(X)$ and $Q(X)$ have the expected value at $b$, and, therefore, by Lemma $10.3 \mathrm{ac}_{0}(c)$ may be computed from $\mathrm{ac}_{0}(b)$. As there is no extension of the value group, this observation shows that the $\mathcal{L}_{K}$-structure is pinned down.

In the case of $v e=0$, we need a different argument. We prove uniqueness first and then show existence. In the course of showing that the $\mathcal{L}_{\mathrm{vdf}} K^{\text {-structure is }}$ uniquely determined, we will show that every new element of the residue field is a $\mathcal{O}_{K} / \mathfrak{m}_{K}$-rational function of $\mathrm{ac}_{0}(b)$ and its conjugates under integral powers of $\sigma$. Thus, included in the proof of the uniqueness of the $\mathcal{L}_{\mathrm{vdf}} K^{\text {-structure is a proof of }}$ the uniqueness of the $\mathcal{L}_{K}$-structure.

As in the proof of Lemma 10.5, it suffices to work with $\sigma$ and $\sigma^{-1}$ polynomials rather than $D$-polynomials. Substituting $e^{-1}(\sigma-\mathrm{id})$ for the operator $D$ and abusing notation, we regard $P$ as a $\sigma$-polynomial.

Let $d:=\operatorname{ord} P$. Let $K^{(n)}:=K\left(b, \ldots, \sigma^{n}(b)\right)$ for $n \geq 0, K^{(-1)}:=K$, and $L_{+}:=\bigcup_{n \in \omega} K^{(n)}$.

We show now by induction on $n$ that the $\mathcal{L}_{\mathrm{vdf} K^{-}}$-structure on $K^{(n)}$ is uniquely determined. When $n \leq d$, then every element of $K^{(n)}$ may be expressed as quotient of $\sigma$-polynomials simpler than $P$. Just as in the case of $v e>0$, the $\mathcal{L}_{\text {vdf } K}$-structure is determined on $K^{(n)}$.

The case of $n=d+j$ with $j \geq 0$ requires some work. Write $P(X)=$ $F\left(X, \sigma(X), \ldots, \sigma^{d}(X)\right)$ with $F\left(X_{0}, \ldots, X_{d}\right) \in \mathcal{O}_{K}\left[X_{0}, \ldots, X_{d}\right]$. Set $G(Y):=F\left(b, \ldots, \sigma^{d-1}(b), Y\right)$. Then $G(Y)$ is a minimal polynomial of $\sigma^{d}(b)$ over $K^{(d-1)}$. By hypothesis, $\pi(b)$ is a simple root of $\pi(G)(Y)$. As $\sigma$ induces an automorphism of the residue field, $\sigma^{j}(b)$ is a simple root of $\pi\left(\sigma^{j}(G)\right)(Y)$ for each $j \geq 0$. Thus, $K^{(d+j)}$ is contained in the strict henselization of $K^{(d)}$ for each $j \geq 0$. Moreover, for each $j \geq 0$, as a valued field the extension $K^{(d+j)} / K^{(d+j-1)}$ is characterized over $K^{(d+j-1)}$ and $k_{K}(\langle\pi(b)\rangle)$ by the conditions that $\sigma^{j}(b)$ is the unique solution to $\sigma^{j}(G)(Y)=0$ and $\pi(Y)=\sigma^{j}(\pi(b))$.

To get all of $L$ we need to consider $\bigcup_{n=0}^{\infty} \sigma^{-1}\left(L_{+}\right)$. We do this by working with $H(Y):=F\left(Y, \sigma(b), \ldots, \sigma^{d}(b)\right)$ instead of $G(Y)$.

For existence, pick some realization $\bar{b} \models p$. Give $K^{\prime}:=K\left(X_{0}, \ldots, X_{d-1}\right)$ the structure of a valued field with residue field contained in $\mathcal{O}_{K} / \mathfrak{m}_{K}(\langle\bar{b}\rangle)$ by defining $v\left(\sum p_{\alpha} X^{\alpha}\right):=\min \left\{v\left(p_{\alpha}\right)\right\}$ and $\pi\left(X_{i}\right):=\sigma^{i}(\bar{b})$. It is a routine matter to check that this defines a valuation.

Let $L$ be a strict henselization of $K^{\prime}$. Let $y \in \mathcal{O}_{L}$ with $F\left(X_{0}, \ldots, X_{d-1}, y\right)=0$ and $\pi(y)=\sigma^{d}(\bar{b})$. Note that Hensel's Lemma guarantees the existence of $y$.

Define $\tilde{\sigma}: K^{\prime} \rightarrow L$ by $\left.\tilde{\sigma}\right|_{K}:=\sigma, \tilde{\sigma}\left(X_{i}\right):=X_{i+1}$ for $0 \leq i<d-1$ and $\tilde{\sigma}\left(X_{d-1}\right):=$ $y$. By the irreducibility of $P, X_{1}, \ldots, X_{d-1}, y$ are algebraically independent over $K$. 
Hence, a unique field homomorphism is specified by the above conditions. Using the facts that $v y \geq 0$ and $\sigma$ preserves the valuation on $K$, we see that the image of $\tilde{\sigma}$ on $\mathcal{O}_{K^{\prime}}$ is contained in $\mathcal{O}_{L}$. Thus, by the universal property of the strict henselization, there is an extension of $\tilde{\sigma}$ to $\mathcal{O}_{L}$. The extension need not be unique, but on the (inversive) difference field generated by $X_{0}$ over $K, K\left(\left\langle X_{0}\right\rangle\right)$, it is unique as it is the only such map lifting $\sigma$. Likewise, we find a map $\tilde{\sigma}^{-1}: \mathcal{O}_{L} \rightarrow \mathcal{O}_{L}$ which when restricted to $\mathcal{O}_{K\left(\left\langle X_{0}\right\rangle\right)}$ is an inverse to $\tilde{\sigma}$. As both $\tilde{\sigma}$ and $\tilde{\sigma}^{-1}$ preserve the valuation ring, they must preserve the valuation itself.

As remarked in the introduction, the main reason for introducing angular component functions is to deal with radical extensions. We do this with the next two lemmata.

Lemma 10.7 If $K$ is a valued $D$-field and $\pi: \mathcal{O}_{K} \rightarrow k_{K}$ is surjective, then for any $b \in K^{\times}$there is some $\epsilon \in K$ satisfying $v \epsilon=v b$ and $\mathrm{ac}_{0}(\epsilon)=1$ (and, therefore, $v D \epsilon>v \epsilon)$.

Proof As $\pi$ is surjective, there is some $c \in \mathcal{O}_{K}^{\times}$with $\pi(c)=\operatorname{ac}_{0}(b)^{-1}$. Set $\epsilon:=c \cdot b$.

Lemma 10.8 If $K$ is a valued $D$-field and $\eta \in K^{\times}$with

- $\operatorname{ac}_{0}(\eta)=1$,

- $\operatorname{tp}(v(\eta)) \vdash n \mid x$, and

- $v(\eta) \notin m v K$ for all $m<n$ with $m \mid n$,

then the field $K(\langle\epsilon\rangle)$ where $\epsilon^{n}=\eta$ has a unique $\mathcal{L}_{K}$-structure with $\mathrm{ac}_{0}(\epsilon)=1$.

Proof That the valuation structure on $K(\epsilon)$ is determined is common knowledge. Moreover, since $\epsilon$ has the expected value for every polynomial over $K$ of degree less than $n$, the angular component structure is pinned down. There is no residue extension, so we need not worry about the possible new $\mathcal{L}_{K}$-relations. The new elements of the value group all lie in the group generated by $v K$ and $v(\epsilon)$ which is definable from $v(\eta)$.

In the case that $e=0$, there is nothing more to do as in this case $D \epsilon \in K(\epsilon)$. We assume now that $e \neq 0$.

As $\sigma$ must preserve the valuation, we see that $\sigma(\epsilon)=\omega \cdot \epsilon$ where $v(\omega)=0$. As $\sigma$ commutes with $\mathrm{ac}_{0}$, we see that $\pi(\omega)=\mathrm{ac}_{0}(\omega)=1$. The image of $\epsilon$ under $\sigma$ must be a root to $X^{n}=\sigma(\eta)$. Hence, $\omega$ is a root to $X^{n}=\frac{\sigma \eta}{\eta}$. Note that $\pi\left(\frac{\sigma(\eta)}{\eta}\right)=1$. As the residue characteristic is zero, there is a unique element $\tilde{\omega}$ of the henselization of $K(\epsilon)$ with $\pi(\tilde{\omega})=1$ and $\tilde{\omega}^{n}=\frac{\sigma(\eta)}{\eta}$. Thus, by the universal property of the henselization, there is a unique embedding of $K(\langle\epsilon\rangle)$ into its henselization compatible with $\left.\sigma\right|_{K}$, $\sigma(\epsilon)^{n}=\sigma(\eta)$, and $\mathrm{ac}_{0}(\sigma(\epsilon))=1$.

The next lemma is the analogue of the above lemma for values linearly independent from the value group of the base field.

Lemma 10.9 If $K$ is a valued $D$-field, $p(x) \in S_{1, \Gamma}(v K)$, and $p(x) \vdash\{n x \neq$ $\left.v(b): n \in \mathbb{Z}_{+}, b \in A\right\}$, then $K(\epsilon)$ has a unique $\mathcal{L}_{K}$-structure with $D \epsilon=0, \mathrm{ac}_{0}(\epsilon)=1$, and $v(\epsilon) \models p$.

Proof Lemma 7.8 of [12] shows that $K(\epsilon)$ has a unique $\mathcal{L}_{\text {vdf } K}$-structure with $v \epsilon \mid=p$ and $D \epsilon=0$. As there is no extension of the residue field and $p$ determines the 
extension on the value group, this structure is also determined. As every polynomial over $K$ has the generic valuation at $\epsilon$, the expansion to $\mathcal{L}_{\mathrm{vdf} K}^{\mathrm{ac}}$ is also determined.

We proceed now to prove Theorem 6.3.

\section{Proof of Main Theorem:}

We follow the strategy and arguments of [12] quoting the above lemmata in some places to guarantee that the arguments work in $\mathcal{L}$ with ve $\geq 0$.

We remind the reader that we are given two $\aleph_{1}$-saturated $\mathcal{L}$-structures $M_{1}$ and $M_{2}$, a countable substructure $A \subseteq M_{1}$, an $\mathcal{L}$-embedding $f: A \hookrightarrow M_{2}$ and some element $a \in M_{1}$. We are charged with finding an extension of $f$ to an $\mathcal{L}$-embedding of $A_{1}(\langle a\rangle)$ into $M_{2}$.

We extend the embedding $f: A \hookrightarrow M_{2}$ in stages.

We find a countable elementary submodel $N_{1} \prec M_{1}$ with $A(\langle a\rangle) \subseteq N_{1}$.

We extend $f$ so that $\pi\left(\mathcal{O}_{A}\right)=k_{N_{1}}$ using Lemma 10.6. That is, if $\bar{b} \in k_{N_{1}} \backslash$ $\pi\left(\mathcal{O}_{A}\right)$, then we choose $P(X) \in \mathcal{O}_{A}\langle X\rangle_{D}$ so that T.deg $\pi(P)=\mathrm{T} \cdot \operatorname{deg}(P)$ and $\pi(P)$ is a minimal $D$-polynomial for $b$ over $\mathcal{O}_{A} / \mathfrak{m}_{A}$. As we have assumed quantifier elimination for the residue field, we may extend $f$ to $b$. As $N_{1}$ is $D$-henselian, there is some $\tilde{b} \in \mathcal{O}_{N_{1}}$ with $\pi(\tilde{b})=\bar{b}$. Likewise, there is some $\tilde{c} \in \mathcal{O}_{M_{2}}$ with $\pi(\tilde{c})=f(\bar{b})$.

As the residue characteristic is zero, DHL applies to $P$ at $\tilde{b}$, and, hence, also to $f(P)$ at $\tilde{c}$. Let $b \in \mathcal{O}_{N_{1}}$ with $P(b)=0$ and $\pi(b)=\bar{b}$ and $c \in \mathcal{O}_{M_{2}}$ with $f(P)(c)=0$ and $\pi(c)=f(\bar{b})$. By Lemma 10.6 there is an extension of $f$ to $A(\langle b\rangle)$ determined by $b \mapsto c$.

We then extend $f$ so that $v A=\Gamma_{N_{1}}$. There are two different steps involved with this kind of extension.

If $\gamma \in \Gamma_{N_{1}}$ but $n \gamma \notin v A$ for all $n \in \mathbb{Z}_{+}$, then by Lemma 10.9 we may extend $f$ so that $\gamma=v b$ for some $b$ in the domain.

In the other case, take $n$ minimal with $n \gamma \in v A$. If $n=1$, there is nothing to do. Otherwise, since $\mathcal{O}_{A} / \mathfrak{m}_{A}=k_{N_{1}}$ and $\pi$ is surjective on $\mathcal{O}_{N_{1}}$, by Lemma 10.7 there is some $\eta \in A$ with $v(\eta)=n \gamma$ and $\operatorname{ac}_{0}(\eta)=1$. Let $\tilde{\epsilon} \in N_{1}$ with $v(\tilde{\epsilon})=\gamma$ and $\operatorname{ac}_{0}(\tilde{\epsilon})=1$. Applying Hensel's Lemma to $X^{n}-\frac{\eta}{\tilde{\epsilon}^{n}}$ at 1 we find that there is some $\epsilon \in N_{1}$ with $\epsilon^{n}=\eta$ and $\operatorname{ac}_{0}(\epsilon)=1$. Likewise, there is some $\zeta \in M_{2}$ with $\zeta^{n}=f(\eta)$ and $\operatorname{ac}_{0}(\zeta)=1$. By Lemma 10.8 we may extend $f$ to $A(\langle\epsilon\rangle)$ by sending $\epsilon \mapsto \zeta$.

We have reduced to the case that $N_{1}$ is an immediate extension of $A$. If one is willing to carefully examine the proofs in section 7.2 of [12], then one sees that the hypothesis that $A$ has enough constants is not used when dealing with algebraic extensions or when dealing with extensions for which $D(b)$ is rational over $b$. This shows that it is possible to extend the embedding inside $N_{1}$ so that $A$ has enough constants. However, as I have disavowed such tests of the readers' patience, we must follow a different route.

We will find a countable unramified extension $A^{(2)}$ of $A$ on which an extension of $f$ is defined and which has enough constants. We then take $N_{1} \preceq N_{1}^{(2)} \prec M_{1}$ a countable model with $A^{(2)} \subseteq N_{1}^{(2)}$. We repeat the above arguments extending so that $N_{1}^{(2)}$ is an immediate extension of the domain of $f$. We then find $A^{(3)}$, a countable unramified extension of $A^{(2)}$ on which an extension of $f$ is defined and which has enough constants, and so on. Eventually, we let $N$ be the direct limit of the $N_{1}^{(i)}$ 's and we will have that

- $N$ is a countable elementary submodel of $M_{1}$ (and, therefore, has enough constants), 
- $N$ is an immediate extension of the domain of $f$, and

- $a \in N$.

To extend $f$ so that the domain has enough constants, take $\gamma \in \Gamma_{A}$. As $M_{1}$ is $D$-henselian and $\aleph_{1}$-saturated, we can find $\epsilon \in M_{1}$ with $D \epsilon=0, v(\epsilon)=\gamma$, and $\mathrm{ac}_{0}(\epsilon) \notin k_{A}^{a l g}$. One computes easily that every polynomial over $A$ has the expected valuation at $\epsilon$ so that the $\mathcal{L}_{A}$-structure on the unramified extension $A(\langle\epsilon\rangle)$ is determined by $p(x):=\operatorname{tp}\left(\operatorname{ac}_{0}(\epsilon) / k_{A}\right)$. Using the fact that $M_{2}$ is an $\aleph_{1}$-saturated $D$-henselian field we find $\zeta \in M_{2}$ with $v(\zeta) \models f\left(\operatorname{tp}\left(\gamma / \Gamma_{A}\right)\right), D \zeta=0$, and $\operatorname{ac}_{0}(\zeta) \models$ $f(p)$.

From now on, we may assume that $A$ has enough constants and that $N$ is an immediate extension of $A$. Given Lemma 10.4, the proof for this last step is (almost) the same as the proof in [12]. The sole use of the hypothesis $v(e)>0$ in the original proof is cosmetic: in the last paragraph of Lemma 7.47 the fact that $\mathcal{D}_{e}(R)$ is henselian is used. However, when $v(e)=0$, this ring is isomorphic to $R \times R$ so that one should apply the universal property of the Cartesian product and of the henselization to find the unique extension $\psi$.

Proposition 7.51 of [12] shows that there is an extension of $f$ to an $\mathcal{L}_{\text {vdf } K^{-}}$ embedding of $N$ into $M_{2}$. By Lemma 10.4, this is also an $\mathcal{L}_{K}$-embedding.

\section{Eliminating the leading terms}

The angular component functions and leading term structures used in this paper clutter our results. In this section we note that the results of the previous section suffice for a complete axiomatization of the theory of a $D$-henselian field of characteristic zero.

To begin, we note Theorem 6.3 implies a complete axiomatization in $\mathcal{L}_{\mathrm{vdf}}$.

Theorem 11.1 Let $K$ be a D-henselian field of characteristic zero. Then the theory of $K$ in $\mathcal{L}_{\mathrm{vdf}}$ is determined by the theory of D-henselian fields of characteristic zero, the atomic theory of $K$, the theory of the value group and the theory of $\bigcup_{n \in \omega} R_{n}(K)$ considered as a many sorted structure.

Proof: As the statement of this theorem is absolute, we may and do assume GCH. Let $K^{\prime} \succ K$ be a saturated elementary extension of $K$. Let $\chi: v K^{\prime} \rightarrow\left(\left(K^{\prime}\right)^{D}\right)^{\times}$ be a section of the valuation on $K^{\prime}$. If the residue characteristic of $K$ is $p$, define $\operatorname{ac}_{n}(x):=\frac{x}{\chi(v x)}+p^{n} \mathfrak{m}_{K^{\prime}}$ for $x \in\left(K^{\prime}\right)^{\times}$and $n \in \omega$. If the residue characteristic is zero, set $\operatorname{ac}_{0}(x):=\frac{x}{\chi(v x)}+\mathfrak{m}_{K^{\prime}}$ for $x \in\left(K^{\prime}\right)^{\times}$. With these functions $K^{\prime}$ is a $D$ henselian field of characteristic zero with a system of angular component functions. By Theorem 6.3, the theory of $K^{\prime}$ in $\mathcal{L}_{\mathrm{vdf}}^{\mathrm{ac}}$ is determined by this fact and the theory of its value group and of $\bigcup R_{n}\left(K^{\prime}\right)$. Hence, the theory of its reduct to $\mathcal{L}_{\mathrm{vdf}}$ is determined by the same things and thus, the same is true of $K$.

If we specialize $K$, then the statement of Theorem 11.1 becomes cleaner.

Corollary 11.2 The completions of the theory of D-henselian fields of equicharacteristic zero are determined by specifying the atomic theory, the theory of the residue field, and the theory of the value group. 
Remark 11.3 The generalized power series construction of [12] shows that we may take any $D$-linearly closed field of characteristic zero and choose for $e$ any element of non-negative valuation in the generalized power series ring.

Corollary 11.4 Let $p$ be a rational prime. Let $k$ be a field of characteristic $p$ having no degree $p$ extensions. Let $K:=W_{p^{\infty}}(k)\left[\frac{1}{p}\right]$ be the field of fractions of the Witt vectors over $k$. Let $\sigma: K \rightarrow K$ be the relative Frobenius. Let $v: K \rightarrow \mathbb{Z} \cup\{\infty\}$ be the $p$-adic valuation on $K$. Then the theory of $(K, v, \sigma)$ is axiomatized by saying that

- the function $D(x):=\sigma(x)-x$ makes $K$ into a D-henselian field of characteristic zero with $e=1$,

- the residue field satisfies $\operatorname{Th}(k)$,

- the value group is a Z-group with least positive element $v(p)$, and

- $D(x) \equiv x^{p}-x(\bmod p)$ for $x \in \mathcal{O}_{K}$.

Moreover, we have quantifier elimination relative to the residue field in $\mathcal{L}_{\mathrm{vdf}}\left(\left\{\mathrm{ac}_{n}\right\}_{n \in \omega}\right)$ augmented by divisibility predicates on the value group. In particular, when $k=$ $k^{a l g}$, we have absolute quantifier elimination.

Proof The rings $R_{n}(K)$ are definably isomorphic to $W_{p^{n}}(k)$ which are themselves bi-interpretable with $k$.

The reader may substitute finitely ramified extensions of $W_{p^{\infty}}(k)\left[\frac{1}{p}\right]$ where $k$ is an algebraically closed field of characteristic $p$ and powers of the relative Frobenius in the above corollary to obtain other axiomatizations of concrete theories of standard valued difference fields.

Remark 11.5 The $\mathcal{L}_{\mathrm{vdf}}$-structure on $W_{p \infty}\left(\mathbb{F}_{p}^{a l g}\right)\left[\frac{1}{p}\right]$ is an expansion of the Teichmüller structure considered in [8] as the Teichmüller representives are defined by the equation $\sigma(x)=x^{p}$.

Remark 11.6 The standard angular component functions corresponding to the section $n \mapsto p^{n}$ are (existentially) definable on $\mathbb{Q}_{p}$ [7]. It follows that they are (existentially) definable on the Witt vectors of $\mathbb{F}_{p}^{a l g}$ by $y=\mathrm{ac}_{n}(x) \Leftrightarrow(\exists z)[\sigma(z)=$ $\left.z \wedge \mathrm{ac}_{n}(z)=z \wedge v(x)=v(z)\right]$. Thus, the Witt vectors are model complete in $\mathcal{L}_{\text {vdf }}$.

Remark 11.7 There are $D$-henselian fields of mixed characteristic in which the residue field is a differential field. Since we must require the residue field to be $D$-linearly closed, the residue fields necessarily have infinite degree of imperfection.

\section{References}

[1] J. Ax and S. Kochen, Diophantine problems over local fields. III. Decidable fields, Ann. of Math. (2) 83 (1966), 437-456.

[2] Ş. Basarab and F.-V. Kuhlmann, An isomorphism theorem for Henselian algebraic extensions of valued fields, Manuscripta Math. 77 (1992), no. 2-3, 113-126.

[3] L. Bélair, Types dans les corps valus munis d'applications coefficients, Illinois J. Math. 43 (1999), no. 2, 410-425.

[4] L. Bélair and A. Macintyre, L'automorphisme de Frobenius des vecteurs de Witt, C. R. Acad. Sci. Paris Sér. I Math. 331 (2000), no. 1, 1-4.

[5] L. Bélair, A. Macintyre, and T. Scanlon, The relative Frobenius, in preparation.

[6] S. Bosch, W. Lütkebohmert, M. Raynaud, Néron Models, Ergibnisse der Mathematik und ihrer Grenzgebiete (3), 21, Springer-Verlag, Berlin (1990). 
[7] J. Denef, p-adic semi-algebraic sets and cell decomposition, J. Reine Angew. Math. 369 (1986), $154-166$.

[8] L. van den Dries, On the elementary theory of rings of Witt vectors with a multiplicative set of representatives for the residue field, Manuscripta Math. 98 (1999), no. 2, 133-137.

[9] W. Hodges, Model Theory, Encyclopedia of Mathematics and its Applications, 42, Cambridge University Press, Cambridge (1993).

[10] F.-V. Kuhlmann, Quantifier elimination for Henselian fields relative to additive multiplicative congruences, Israel J. Math. 85 (1994), no. 1-3, 277 - 306.

[11] A. Macintyre, On definable subsets of p-adic fields, J. Symbolic Logic 41 (1976), no. 3, 605-610.

[12] T. Scanlon, A model complete theory of valued D-fields, J. Symbolic Logic 65 (2000), no. 4, $1758-1784$.

[13] T. Scanlon, Model theory of valued D-fields, Ph. D. thesis, Harvard University, May 1997. 